\title{
Poliquetos bentónicos submareales de fondos blandos de la región de Aysén, Chile: Clados Amphinomida, Eunicida, Spionida, Sabellida y Scolecida (Annelida, Polychaeta)*
}

\author{
Nicolás Rozbaczylo ${ }^{1}$, Rodrigo A. Moreno ${ }^{1}$ \& Oscar Díaz-Díaz ${ }^{2}$ \\ ${ }^{1}$ Departamento de Ecología, Facultad de Ciencias Biológicas, Pontificia Universidad Católica de \\ Chile, Casilla 114-D, Santiago, Chile \\ ${ }^{2}$ Instituto Oceanográfico de Venezuela, Universidad de Oriente, Cumaná, Venezuela
}

\begin{abstract}
RESUMEN. Se presentan los resultados del análisis taxonómico de los poliquetos bentónicos submareales de los clados Amphinomida, Eunicida, Spionida, Sabellida y Scolecida obtenidos durante los cruceros de investigación Cimar-7 y Cimar-8 Fiordos. Se incluyen observaciones morfológicas e ilustraciones, así como sinonimia y distribución geográfica de cada especie. Las muestras fueron obtenidas en el mar interior de la región de Aysén, Chile, en fondos de limo-arcilla, mediante una rastra Agassiz, entre 20 y $444 \mathrm{~m}$ de profundidad. En ambos cruceros se recolectaron 79 ejemplares del clado Eunicida, distribuidos en seis especies agrupadas en seis géneros y cinco familias, dos ejemplares de los clados Amphinomida y Spionida de las especies Pareurythoe chilensis (Kinberg), y Chaetopterus cf. variopedatus (Renier), respectivamente; 13 ejemplares del clado Sabellida se distribuyen en cuatro especies agrupadas en cuatro géneros y tres familias, y 187 ejemplares del clado Scolecida corresponden a tres especies agrupadas en dos géneros y una familia. Se recolectó un total de tres especies de Maldanidae (20\% de abundancia total en las muestras), dos especies de Lumbrineridae y Serpulidae (13,3\%), y una especie de Amphinomidae, Dorvilleidae, Eunicidae, Oenonidae, Onuphidae, Chaetopteridae, Sabellariidae y Sabellidae (6,7\%), respectivamente. Durante el crucero Cimar-7 se recolectaron 178 ejemplares, destacándose Maldane sarsi antarctica Arwidsson con una abundancia del 73\%. En el crucero Cimar-8 se recolectaron 105 ejemplares, destacándose Maldane chilensis Hartmann-Schröder con una abundancia del 50,5\%. Con estos resultados llega a 91 el número total de especies de poliquetos bentónicos registrados en la región de Aysén.
\end{abstract}

Palabras clave: Polychaeta, aguas templadas frías, Provincia Magallánica, Pacífico suroriental.

\section{Benthic subtidal polychaetes on soft bottoms in Aysén, Chile: Clades Amphinomida, Eunicida, Spionida, Sabellida and Scolecida (Annelida, Polychaeta)*}

\begin{abstract}
We present results from a taxonomic study of subtidal benthic polychaetes belonging to the Amphinomida, Eunicida, Spionida, Sabellida and Scolecida clades, obtained during the Cimar-7 and Cimar-8 Fjords research cruises. We include morphological observations and illustrations, synonymy and the geographical distribution for each species. Samples were obtained in the Aysén region ( $43^{\circ} 45^{\prime}$ S- $46^{\circ} 28^{\prime} \mathrm{S}$ ), on the southeastern Pacific coast of Chile, from silt-clay bottoms using an Agassiz trawl between 20 and $444 \mathrm{~m}$ depth. During the two cruises we collected 79 specimens from the Eunicida clade (corresponding to six species, grouped into six genera and five families); two specimens of the species Pareurythoe chilensis (Kinberg) and Chaetopterus cf. variopedatus (Renier) from the Amphinomida and Spionida clades, respectively; 13 specimens from the Sabellida clade (four species, grouped into four genera and three families); and 187 specimens from the Scolecida clade (three species grouped into two genera and one family). We collected a total of three species of Maldanidae (20\% of the total sampled abundance), two species of Lumbrineridae and Serpulidae (13.3\%), and one species each of Amphinomidae, Dorvilleidae, Eunicidae, Oenidae, Onuphidae, Chaetopteridae, Sabellariidae and Sabellidae (6.7\%), respectively. During the Cimar-7 cruise we collected a total of 178 polychaete specimens, noteably
\end{abstract}

Autor corresponsal: Nicolás Rozbaczylo (nrozbaczylo@bio.puc.cl)

* Trabajo presentado en el XXV Congreso de Ciencias del Mar de Chile y XI Congreso Latinoamericano de Ciencias del Mar (COLACMAR), realizados en Viña del Mar, entre el 16 y 20 de mayo de 2005. 
Maldane sarsi antarctica Arwidsson which represented $73 \%$ of total abundance. During the Cimar- 8 cruise we collected 105 polychaetes; Maldane chilensis Hartmann-Schröder represented 50.5\% of total sampled species abundance. Our findings increase the total number of benthic polychaete species currently recorded for the Aysén region to 91 species.

Key words: Polychaeta, cold temperate waters, Magellanic Province, southeastern Pacific.

\section{INTRODUCCIÓN}

A nivel mundial, el clado Amphinomida incluye dos familias: Amphinomidae y Euphrosinidae, con cerca de 200 especies agrupadas en 25 géneros (Rouse \& Pleijel, 2001). Este clado ha sido considerado el grupo hermano putativo de Eunicida, por presentar una similitud estructural del estomodeo (Rouse \& Fauchald, 1997). El clado Eunicida se encuentra representado en el mundo por siete familias y más de 800 especies (Rouse \& Pleijel, 2001). La evidencia de monofilia la constituye la presencia de un complejo aparato mandibular esclerotizado y/o mineralizado (Glasby et al. 2000). El clado Spionida incluye ocho familias y más de 600 especies y se define por la presencia de un par de palpos peristomiales acanalados, órganos nucales que forman una proyección posterior, nefridios excretores anteriores y órganos segmentales posteriores (Rouse, 2000c). El clado Sabellida incluye cinco familias y más de 1100 especies, y se define por la presencia del prostomio fusionado con el peristomio y pérdida de los labios peristomiales (Rouse, 2000b). El clado Scolecida agrupa a nueve familias y más de 800 especies (Rouse \& Pleijel, 2001) y se define por la presencia de sólo dos autapomorfías, la presencia de parápodos con ramas similares y la presencia de dos o más pares de cirros pigidiales (Rouse, 2000a).

Las especies de los cinco clados se caracterizan por presentar una gran variedad de estrategias tróficas, incluyendo carnívoros, herbívoros, depositívoros, comensales y parásitos (Glasby et al. 2000; Rouse \& Pleijel, 2001).

En la costa del Pacífico suroriental frente a Chile, se han registrado hasta ahora nueve especies del clado Amphinomida, 57 del clado Eunicida, 47 especies del clado Spionida, 57 especies clado Sabellida y 70 especies del clado Scolecida (Rozbaczylo, 1985). La información de estos clados para la zona austral de Chile en la región de Aysén proviene principalmente de expediciones extranjeras que visitaron la costa hace más de 40 años (Wesenberg-Lund, 1962; Hartmann-Schröder, 1965) y de un estudio más reciente de Cañete et al. (1999) en el fiordo Aysén.
Con la realización de los programas de investigación Cimar-7 y Cimar-8 Fiordos, entre los años 2001 y 2002 en la región de Aysén, se ha podido incrementar la información de áreas remotas y de difícil acceso de la costa de Chile, contribuyendo a generar nuevo conocimiento científico sobre la fauna de poliquetos bentónicos de la región (Rozbaczylo et al., 2005; Rozbaczylo et al., 2006). El objetivo del presente trabajo es dar a conocer las especies de poliquetos de los clados Amphinomida, Eunicida, Spionida, Sabellida y Scolecida recolectadas en muestras bentónicas obtenidas durante los cruceros Cimar-7 y Cimar-8 Fiordos en la región de Aysén y entregar antecedentes taxonómicos de cada especie.

\section{MATERIALES Y MÉTODOS}

Los poliquetos se obtuvieron como parte de muestras bentónicas recolectadas durante los cruceros Cimar-7 y Cimar-8 Fiordos, desde boca del Guafo $\left(43^{\circ} 45^{\prime} \mathrm{S}\right)$ a estero Elefante $\left(46^{\circ} 28^{\prime}\right.$ 'S), en julio de 2001 , a profundidades entre 20 y $444 \mathrm{~m}$, y desde boca del Guafo a bahía Anna Pink (454'ㄱ), en julio de 2002, a profundidades entre 62 y $345 \mathrm{~m}$, respectivamente, a bordo del AGOR "Vidal Gormaz" (ver tablas y mapas en Rozbaczylo et al., 2005; Rozbaczylo et al., 2006, respectivamente).

Las muestras fueron obtenidas con una rastra Agassiz de 1,4 $\mathrm{m}$ de apertura de boca y $0,5 \mathrm{~m}$ de alto, en fondos de limo-arcilla. Los poliquetos contenidos en cada muestra de sedimento fueron preservados en una solución de formalina en agua de mar al $10 \%$. Para la determinación taxonómica se utilizó los trabajos de Hartmann-Schröder (1962, 1965), Fauchald (1977) y Rozbaczylo (1980). Los taxa fueron agrupados en los clados Amphinomida y Eunicida, según Rouse \& Pleijel (2001) y en Spionida, Sabellida y Scolecida, según Rouse \& Fauchald (1997).

Los ejemplares de cada especie se midieron con un ocular graduado, considerando la longitud total desde el extremo anterior del prostomio hasta el final del cuerpo y el ancho máximo desde el extremo distal de las setas opuestas de cada parápodo. De cada especie existen ejemplares depositados en la 
sala "Colección de Flora y Fauna Profesor Patricio Sánchez Reyes", de la Facultad de Ciencias Biológicas de la Pontificia Universidad Católica de Chile (SSUC), y en el Museo Nacional de Historia Natural, Santiago (MNHNCL-An).

\section{RESULTADOS}

En ambos cruceros se recolectaron 79 ejemplares del clado Eunicida correspondientes a seis especies agrupadas en seis géneros y cinco familias, dos ejemplares de los clados Amphinomida y Spionida pertenecientes a las especies Pareurythoe chilensis (Kinberg) y Chaetopterus cf. variopedatus (Renier), respectivamente, 13 ejemplares del clado Sabellida correspondientes a cuatro especies agrupadas en cuatro géneros y tres familias, y 187 ejemplares del clado Scolecida correspondientes a tres especies agrupadas en dos géneros y una familia. Se recolectaron un total de tres especies de Maldanidae (20\% de abundancia total en las muestras), dos especies de Lumbrineridae y Serpulidae (13,3\%), y una especie de Amphinomidae, Dorvilleidae, Eunicidae, Oenonidae, Onuphidae, Chaetopteridae, Sabellariidae y Sabellidae $(6,7 \%)$, respectivamente. Durante el crucero Cimar-7 se recolectaron un total de 178 ejemplares de poliquetos, destacándose Maldane sarsi antarctica Arwidsson con una abundancia del 73\% (Tabla 1). En el crucero Cimar-8 se recolectaron 105 poliquetos, destacándose Maldane chilensis Hartmann-Schröder con una abundancia del 50,5\% (Tabla 1). Con estos resultados aumenta a 91 el total de especies de poliquetos bentónicos registradas en la región de Aysén.

Tabla 1. Especies de poliquetos bentónicos de los clados Amphinomida, Eunicida Spionida, Sabellida y Scolecida recolectadas en la región de Aysén, durante los cruceros Cimar-7 y Cimar-8 Fiordos, en julio de 2001 y julio de 2002. Para cada especie se presenta el número de ejemplares recolectados en cada crucero y la abundancia relativa $(\%)$. Las abreviaciones indican: A: Amphinomidae; D: Dorvilleidae; Eu: Eunicidae; L: Lumbrineridae; O: Oenonidae; On: Onuphidae; Ch: Chaetopteridae; Sa: Sabellariidae; S: Sabellidae; Se: Serpulidae, y M: Maldanidae.

Table 1. Benthic polychaete species of the clades Spionida, Sabellida y Scolecida, collected during the Cimar-7 and Cimar-8 Fjords cruises at the region of Aysén, July 2001 and July 2002. For each species we provide the number of specimens collected during each cruise and the relative abundance (\% of total). The abbreviations are: A: Amphinomidae; D: Dorvilleidae; Eu: Eunicidae; L: Lumbrineridae; O: Oenonidae; On: Onuphidae; Ch: Chaetopteridae; Sa: Sabellariidae; S: Sabellidae; Se: Serpulidae, and M: Maldanidae.

\begin{tabular}{|c|c|c|c|c|}
\hline Especie & Cimar-7 & Abundancia (\%) & Cimar-8 & Abundancia (\%) \\
\hline Pareurythoe chilensis (A) & 0 & 0 & 2 & 1,9 \\
\hline Schistomeringos chilensis (D) & 1 & 1 & 0 & 0 \\
\hline Eunice pennata $(\mathrm{Eu})$ & 1 & 1 & 4 & 3,8 \\
\hline Eranno chilensis $(\mathrm{L})$ & 20 & 11 & 6 & 5,7 \\
\hline Ninoe leptognatha $(\mathrm{L})$ & 0 & 0 & 4 & 3,8 \\
\hline Notocirrus cf. virginis $(\mathrm{O})$ & 2 & 1 & 0 & 0 \\
\hline Onuphis pseudoiridescens (On) & 20 & 11 & 21 & 20 \\
\hline Chaetopterus cf. variopedatus (Ch) & 1 & 1 & 1 & 1,0 \\
\hline Idanthyrsus armatus (Sa) & 0 & 0 & 3 & 2,9 \\
\hline Fabricinae (S) & 0 & 0 & 1 & 1,0 \\
\hline Apomatus sp. (Se) & 0 & 0 & 4 & 3,8 \\
\hline Paralaeospira levinseni $(\mathrm{Se})$ & 0 & 0 & 5 & 4,8 \\
\hline Asychis amphiglypta (M) & 0 & 0 & 1 & 1,0 \\
\hline Maldane chilensis (M) & 3 & 2 & 53 & 50,5 \\
\hline Maldane sarsi antarctica $(\mathrm{M})$ & 130 & 73 & 0 & 0 \\
\hline Total & 178 & 100 & 105 & 100 \\
\hline
\end{tabular}




\section{CLADO AMPHINOMIDA}

\section{AMPHINOMIDAE Savigny \\ Pareurythoe chilensis (Kinberg, 1857)}

(Figs. 1a-1h)

Eurythoe chilensis Kinberg, 1857: 13; 1858-1910: 35-35, pl. 12, fig. 9.

Pareurythoe chilensis: Hartman, 1948: 45-46, pl. 5, fig. 11; Wesenberg-Lund, 1962: 38; Rozbaczylo, 1985: 46.

Material examinado: Cimar-8: E-43 (1 ejemplar, SSUC 7167), E-46 (1 ejemplar, MNHNCL-An 2041).

Caracterización: el ejemplar de mayor tamaño midió $39 \mathrm{~mm}$ de largo y $3 \mathrm{~mm}$ de ancho. Prostomio globular con dos pares de ojos, los anteriores más grandes que los posteriores (Fig. 1a). Cinco antenas, la media está inserta en la región occipital y es de igual longitud que el par dorsal, las del par ventral son ligeramente más cortas. Carúncula simple que se extiende hasta el segundo setígero. El primer par de branquias, ubicado en el segundo setígero, consiste en dos filamentos bifurcados; a partir del tercer setígero el número de filamentos branquiales se incrementa hasta un máximo de 12 , en arreglo palmeado (Fig. 1b). Notosetas: (1) furcadas, con una de las ramas distalmente redondeadas y con cuatro dientes diminutos (Fig. 1c), (2) furcadas, con una de las ramas largas y aserrada (Fig. 1d), (3) setas arponadas ligeramente aserradas (Fig. 1e) y (4) setas aciculares (hastate) (Fig. 1f). Neurosetas furcadas, similares a las notosetas, pero con el diente secundario mucho más pronunciado y con mayor número de dientes diminutos (Fig. 1g); setas furcadas con dos dientes subdistales (Fig. 1h) y lisas distalmente redondeadas.

Observaciones: las características de los ejemplares examinados coinciden con lo señalado por Hartman (1948). Sin embargo, en los neurópodos se pudo observar algunas setas furcadas con dos dientes subdistales en vez de uno, aunque la aparición de los mismos es errática. Hartman (1948) señala que $P$. chilensis y P. californica (Johnson) se diferencian por la posición del prostomio, que está localizado entre los dos primeros setígeros en la primera y sólo entre el primero en la segunda. Además, el extremo distal de la neuroseta lisa es distalmente redondeado en $P$. chilensis y distalmente truncado en $P$. californica. Según Hartman (1948), los ejemplares determinados por Monro (1930:28-30, fig. 1a-e), proveniente de Tristan da Cunha, corresponderían a otra especie.
Distribución geográfica: Chile, desde Valparaíso (Kinberg, 1857) hasta la región de Aysén (este estudio).

\section{CLADO EUNICIDA}

\section{DORVILLEIDAE Chamberlin Schistomeringos longicornis (Ehlers, 1901) \\ (Figs. 1i-1o)}

Stauronereis longicornis Ehlers, 1901: 150-151, pl. 19, figs. 18-21, pl. 20, figs. 4-6.

Stauronereis rudolphii: Hartmann-Schröder, 1962: 128-129, figs. 148-150.

Dorvillea rudolphii: Wesenberg-Lund, 1962:118.

Schistomeringos longicornis: Jumars, 1974: 107-109, fig. 2; Rozbaczylo, 1985: 126.

Material examinado: Cimar-7: E- 27 (1 ejemplar, SSUC 7168).

Caracterización: el ejemplar examinado, con 52 setígeros, midió $10 \mathrm{~mm}$ de largo y $1 \mathrm{~mm}$ de ancho. Prostomio redondeado anteriormente, con un par de antenas moniliformes con 7 artejos, dos ojos en la base de las antenas y un par de palpos biarticulados (Fig. 1i). Los dos primeros segmentos ápodos. Segmentos setígeros con parápodos relativamente largos, birrámeos (Fig. 1j). Cirros dorsales en todos los parápodos, con cirróforo largo y cirrostilo corto, con una acícula delgada. Neurópodos conspicuos con tres tipos de setas: furcadas (Fig. 1k), capilares con margen aserrado (Fig. 11) y compuestas distalmente falcadas y bífidas, con el artejo distal de al menos tres longitudes diferentes (Fig. 1m-10).

Distribución geográfica: desde Baja California a Columbia Británica (Jumars, 1974); Chile, desde el seno de Reloncaví (Ehlers, 1901) hasta la región de Aysén (Cañete et al. 1999 y este estudio).

\section{LUMBRINERIDAE Malmgren Eranno chilensis (Kinberg, 1865)} (Figs. 2a-2h)

Lumbriconeris chilensis Kinberg, 1865: 569.

Lumbrineris chilensis: Hartmann-Schröder, 1965: 173-175, figs. 145-148; Rozbaczylo, 1985: 118.

Eranno chilensis: Orensanz, 1990: 78.

Material examinado: Cimar-7: E-6 (1 ejemplar, SSUC 7172), E-17A (1 ejemplar, SSUC 7171), E21 (2 ejemplares, SSUC 7174), E-22 (1 ejemplar, SSUC 7173), E-24 (1 ejemplar, SSUC 7169), E-37 (5 

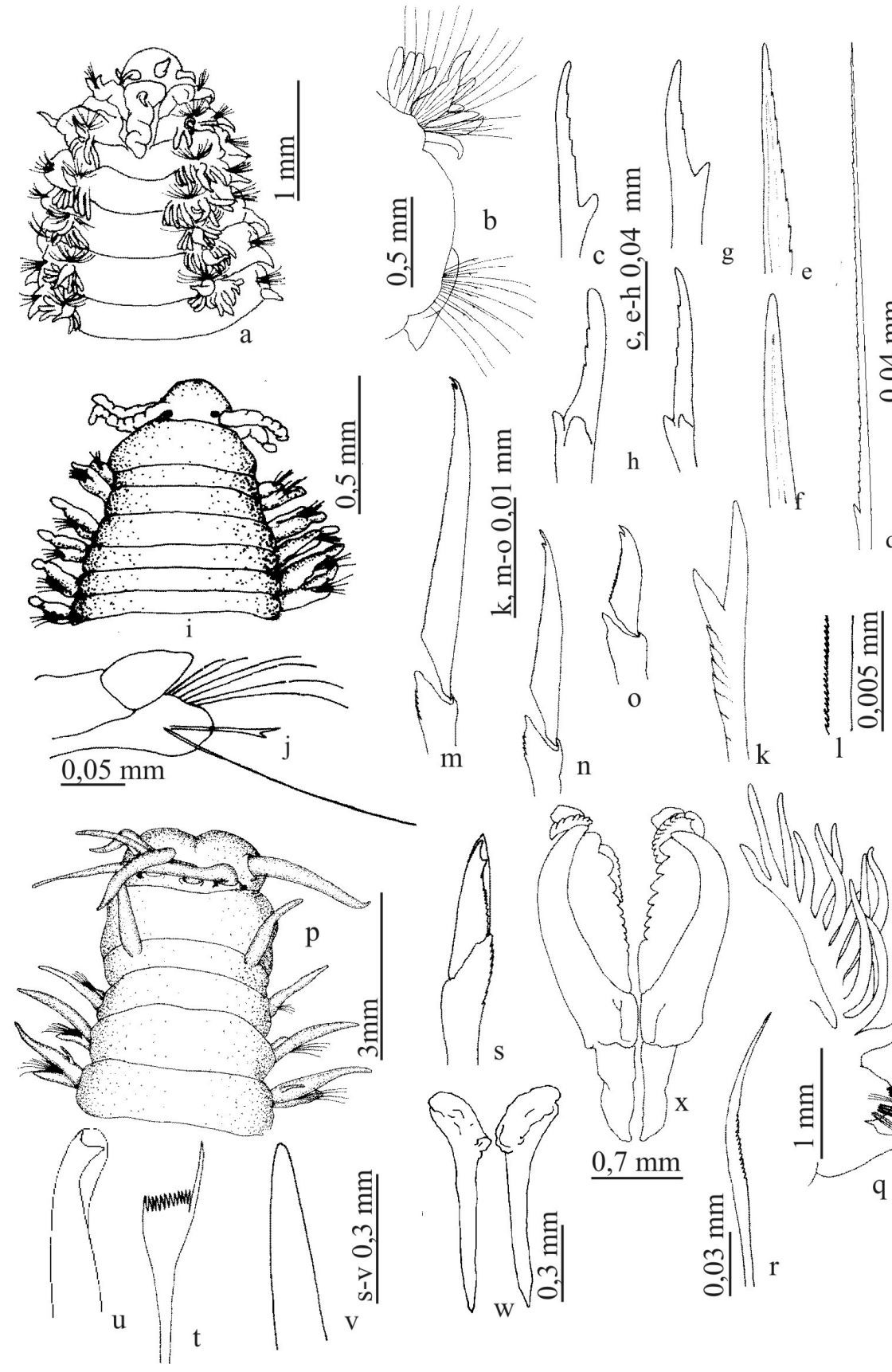

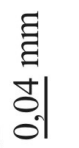

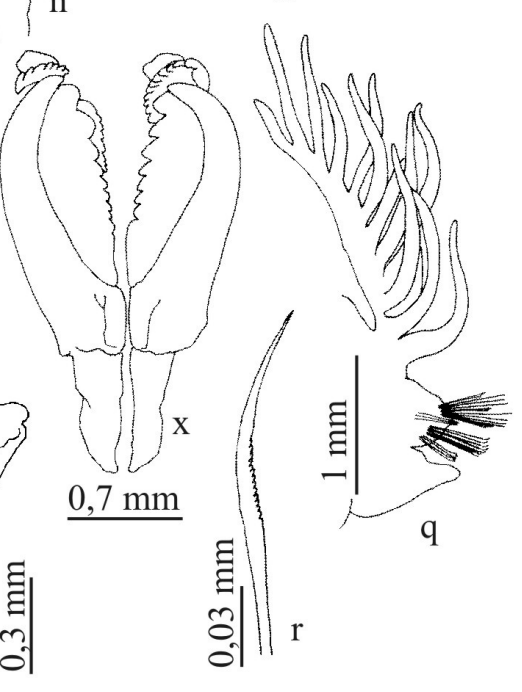

Figura 1. Pareurythoe chilensis: a) extremo anterior, en vista dorsal, b) parápodo medio, c-d) notosetas furcadas, e-f) notosetas arponadas, g) neuroseta furcada, h) neurosetas aciculares; Schistomeringos longicornis: i) extremo anterior, en vista dorsal, $\mathbf{j}$ ) parápodo medio, $k$ ) seta furcada, l) detalle de la seta capilar con margen serrado, mo) setas compuestas; Eunice cf. pennata: p) extremo anterior, en vista dorsal, q) parápodo medio, r) seta superior, s) seta compuesta, t) seta pectinada, u) gancho subacicular, v) acícula, w) mandíbulas, x) complejo maxilar.

Figure 1. Pareurythoe chilensis: a) anterior end in dorsal view, b) middle parapodium, c-d) spurred notosetae, e-f) harpoon notosetae, g) spurred neuroseta, h) acicular neurosetae; Schistomeringos longicornis: i) middle parapodium, k) forked seta, l) detail of the capillary seta with serrated edge, m-o) compound setae; Eunice cf. pennata: $\mathbf{p}$ ) anterior end in dorsal view, q) middle parapodium, r) superior seta, s) compound seta, t) comb seta, u) subacicular seta, v) aciculum, w) mandibles, $x$ ) jaws. 
ejemplares, SSUC 7170); Cimar-8: E-1 (1 ejemplar, SSUC 7176), E-6 (1 ejemplar, SSUC 7177), E-9 (2 ejemplares, SSUC 7179), E-14 (1 ejemplar, SSUC 7181), E-49 (1 ejemplar, 7180), E-53 (1 ejemplar, SSUC 7178), E-54 (1 ejemplar, SSUC 7175), E-72 (1 ejemplar, MNHNCL-An 2035), E-78 ( 1 ejemplar MNHNCL-An 2036).

Caracterización: el ejemplar de mayor tamaño midió $52 \mathrm{~mm}$ de largo y 3,5 $\mathrm{mm}$ de ancho. Prostomio triangular (Fig. 2a), sin ojos. Los primeros dos segmentos son ápodos y aquetos. Parápodos anteriores con el lóbulo setígero redondeado; en los parápodos medios es triangular (Fig. 2b), y en los posteriores es alargado (Fig. 2c). Con ganchos encapuchados multidentados (Fig. 2d-e) desde el primer setígero, más delgados que los de los parápodos medios y posteriores, que son más cortos y anchos y con setas limbadas con el velo estriado (Fig. 2f). Mandíbulas con el borde distal irregular (Fig. 2g). Fórmula maxilar: $\mathrm{I}=1+1$ (falcadas), $\mathrm{II}=5+4, \mathrm{III}=2+2$, IV $=1+1$ (Fig. 2h).

Observaciones: las características de los ejemplares examinados coinciden con las señaladas por Hartmann-Schröder (1965). Orensanz (1990), señala que las diferencias entre las especies de este género son poco claras debido a que muchas de ellas fueron descritas de manera incompleta y por lo tanto el género debe ser revisado.

Distribución geográfica: Chile, desde Coquimbo (Hartmann-Schröder, 1965) hasta la región de Aysén (este estudio).

\section{Ninoe leptognatha Ehlers, 1900}

(Figs. 2i-2n)

Ninoe leptognatha Ehlers, 1900: 215; 1901: 141142, pl. 17, figs. 11-20; Wesenberg-Lund, 1962: 116-117; Orensanz, 1976: 40; 1990: 100-101, pl. 27; Rozbaczylo, 1985: 122; Montiel, Ríos, Mutschke \& Rozbaczylo, 2004: 60.

Material examinado: Cimar-8: E-1 (3 ejemplares, SSUC 7182), E-76 (1 ejemplar, MNHNCL-An 2034).

Caracterización: el ejemplar de mayor tamaño midió $49 \mathrm{~mm}$ de largo y $2 \mathrm{~mm}$ de ancho. Prostomio cónico, corto, con un par de surcos longitudinales (Fig. 2i). Los dos primeros segmentos ápodos y aquetos. Parápodos cortos y redondeados. Con branquias a partir del quinto setígero, hasta el 40-45, con un máximo de seis filamentos (Fig. 2j). Los primeros 35-36 setígeros con setas limbadas (Fig. 2k); a partir del setígero 36-37, en adición a las limbadas, hay ganchos encapuchados multidentados (Fig. 2l) y setas bilimbadas cortas (Fig. 2m). Mandíbulas casi tan largas como la maxila I. Maxilas (Fig. 2n) según la siguiente fórmula: $\mathrm{I}=1+1$ (falcadas), $\mathrm{II}=6+7, \mathrm{III}=$ $5+4, \mathrm{IV}=6+6$, y dos placas laterales pequeñas.

Observaciones: esta especie se asemeja a $N$. falklandica Monro, de la cual difiere por el número máximo de filamentos branquiales: 10-12 para $N$. falklandica y seis para $N$. leptognatha. Por otra parte, Ehlers (1901) y Orensanz (1990) consideran que N. leptognatha y $N$. chilensis están estrechamente relacionadas y que podría tratarse, respectivamente, de morfotipos del noroeste y suroeste de la misma especie.

Distribución geográfica: Chile, desde el estero de Reloncaví (Wesenberg-Lund, 1962) hasta el canal Beagle (Ehlers, 1900).

\section{OENONIDAE Kinberg Notocirrus cf. virginis (Kinberg, 1865)} (Figs. 3a-3h)

Aracoda virginis Kinberg, 1865: 573; 1858-1910: 49, pl. 19, fig. 44.

Notocirrus virginis: Hartman, 1944a: 174; 1948: 97, pl. 13, figs. 8-11; Wesenberg-Lund, 1962: 118; Orensanz, 1974: 391-393, pl. 4; 1976: 41; 1990: 108, pl. 31 , figs. e-i.

Material examinado: Cimar-7: E-24 (1 ejemplar, SSUC 7183), E-25 (1 ejemplar, SSUC 7184).

Caracterización: el ejemplar de mayor tamaño midió $39 \mathrm{~mm}$ de largo y $1,5 \mathrm{~mm}$ de ancho. Prostomio cónico (Fig. 3a), sin ojos. Los dos primeros segmentos ápodos y aquetos. Parápodos con lóbulo setal redondeado (Fig. 3b); con setas limbadas con el margen liso (Fig. 3c) y con el margen aserrado (Fig. 3d); seta acicular ligeramente falcada y distalmente dentada (Fig. 3e); acícula con el extremo distal redondeado (Fig. 3f). Mandíbulas con el margen distal denticulado (Fig. 3g). Maxilas (Fig. 3h), según la siguiente fórmula: $\mathrm{I}=8+9$, con el diente distal más largo y falcado que los siguientes, $\mathrm{II}=7+7, \mathrm{III}=3+4$, $\mathrm{IV}=3+4, \mathrm{~V}=1+1$.

Observaciones: los ejemplares examinados concuerdan bien con las características señaladas por Orensanz (1974) para N. virginis (Kinberg), registrada en la Provincia Magallánica desde Uruguay hasta Cabo Vírgenes, como la ausencia de ojos y la forma y disposición de los dientes maxilares, pero difiere ligeramente en cuanto a las maxilas II-IV. En comparación con $N$. chilensis Schmarda difiere en la 


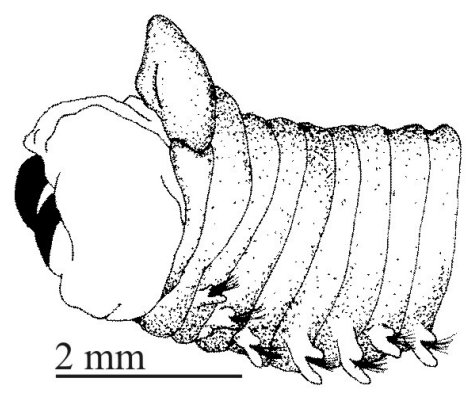

a
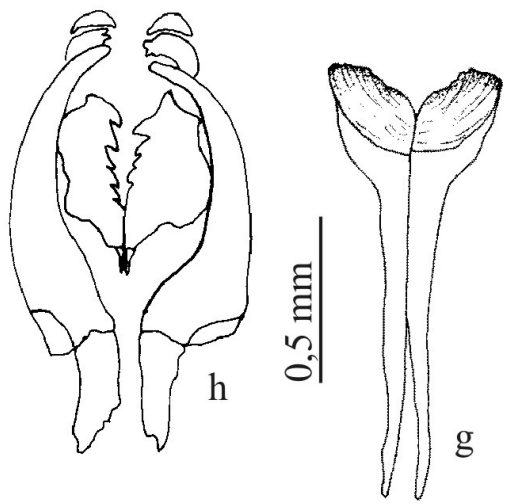

g
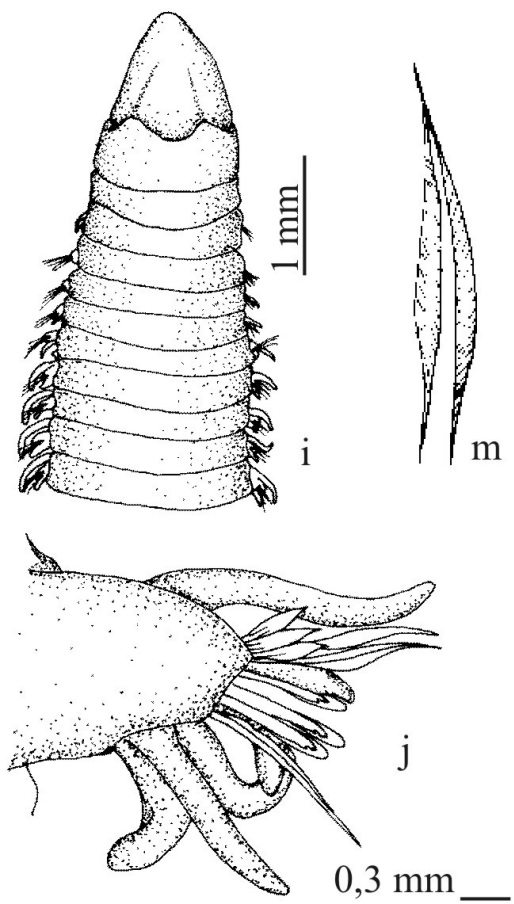
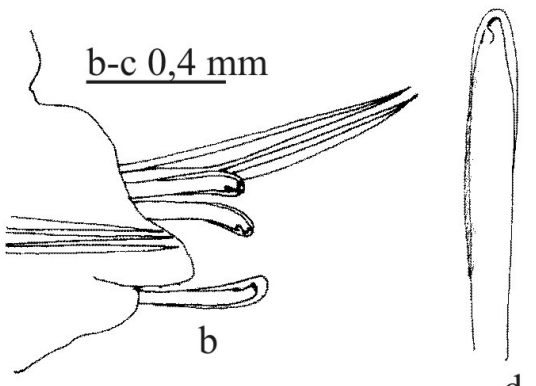

d
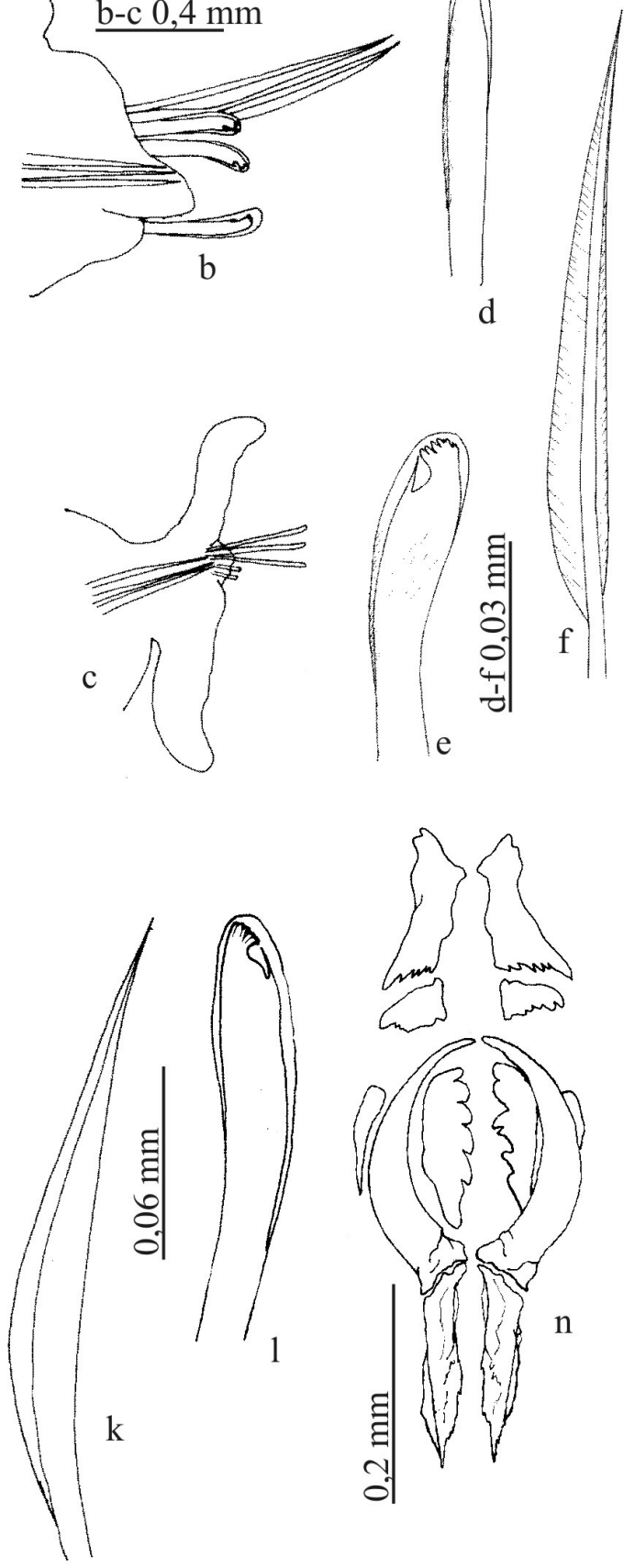

n

Figura 2. Eranno chilensis: a) extremo anterior, en vista lateral, b) parápodo medio, c) parápodo 146, d-e) ganchos multidentados, f) seta limbada, g) mandíbulas, h) complejo maxilar; Ninoe leptognatha: i) extremo anterior, en vista dorsal, j) parápodo 28 , k) seta limbada, l) gancho multidentado, m) seta bilimbada corta, n) complejo maxilar.

Figure 2. Eranno chilensis: a) anterior end in lateral view, b) middle parapodium, c) parapodium 146, d-e) hooded hooks, f) winged capillary, g) mandibles, h) jaws; Ninoe leptognatha: i) anterior end in dorsal view, j) parapodium 28, k) winged seta, l) hooded hook, m) short winged seta, n) jaws. 
fórmula maxilar, pues de acuerdo con la descripción y figuras de Schmarda (1861: 119), ésta sería I = 3+3, II $=2+11, \mathrm{III}=4+4, \mathrm{IV}=6+6$, y no se señala la maxila V. Además, Schmarda (1861) señala la presencia de un solo tipo seta limbada, aquella con el margen del velo aserrado. Con respecto a $N$. lorum Ehlers, la principal diferencia es la presencia de dos pares de ojos y que el diente distal de la maxila I no es más largo que los siguientes.

Distribución geográfica: desde frente a Uruguay a Cabo Vírgenes (Orensanz, 1990); Chile, en la región de Aysén (este estudio).

\section{EUNICIDAE Savigny \\ Eunice pennata (O.F. Müller, 1776)}

(Figs. 1p-1x)

Nereis pennata Müller, 1776: 217.

Eunice pennata: Monro, 1930: 118-119, fig. 42; Fauvel, 1936: 21-22; Hartman, 1964: 118; 1967: 99; Orensanz, 1975: 93, 95, pl. 3; 1990: 66-68, pl. 17, figs. a-f, text-fig. 18; Hartmann-Schröder, 1983: 267; 1986: 81; Knox \& Cameron, 1998: 60-61, figs. 123-125.

Material examinado: Cimar-7: E-32 (1 ejemplar, SSUC 7185); Cimar-8: E-4 (4 ejemplares, SSUC 7186).

Caracterización: el ejemplar de mayor tamaño, con 123 setígeros, midió $98 \mathrm{~mm}$ de largo y $5 \mathrm{~mm}$ de ancho. Prostomio ligeramente bilobulado (Fig. 1p), con un par de ojos y cinco antenas largas muy débilmente articuladas. Peristomio dividido en dos anillos, el segundo más corto que el primero y con un par de cirros tentaculares dorso-laterales. Parápodos con el cirro dorsal largo y el ventral muy corto. Con branquias desde el tercer setígero; las dos primeras como un filamento simple y corto; a partir del cuarto par comienzan a diferenciarse los filamentos que se disponen en forma palmeada llegando a un máximo de 12 en los setígeros medios (Fig. 1q); éstas se mantienen hasta el setígero 39. Setas superiores simples, con serración corta e inconspicua (Fig. 1r). Setas falcígeras compuestas, distalmente bífidas y encapuchadas (Fig. 1s). Setas pectinadas con, aproximadamente, 11-12 dientes, siendo uno de los laterales muy largo (Fig. 1t). Ganchos subaciculares encapuchados bidentados (Fig. 1u). Acículas claras, distalmente redondeadas (Fig. 1v). Mandíbulas oscuras y distalmente blancas (Fig. 1w). Fórmula maxilar: $\mathrm{I}=1+1, \mathrm{II}=8-9,6+6 ; \mathrm{III}=8+0 ; \mathrm{IV}=7-12 ; \mathrm{V}-\mathrm{VI}$ $=1+1($ Fig. $1 \mathrm{x})$.
Observaciones: Las características de los ejemplares examinados coinciden en general, con las señaladas por Orensanz $(1975,1990)$, existiendo sólo una pequeña diferencia en la fórmula maxilar, aunque la misma parece ser variable. Hartman (1964) señala $\mathrm{II}=10+12,7+6$ o $7+9, \mathrm{III}=8+0$ o $10+0, \mathrm{IV}=4+6$, 9+6; Fauchald (1992) señala II $=6+7, \mathrm{III}=9+0, \mathrm{IV}=$ $6-11$, mientras que Orensanz (1975) señala II $=5+6$, $\mathrm{III}=7+9, \mathrm{IV}=5+7,9+10$; esta última caracterización, sin embargo, difiere bastante de la de Hartman (1964) y Fauchald (1992). E. pennata se asemeja a $E$. magellanica, de la cual se diferencia principalmente por la aparición de la primera branquia (setígero 6), por el número máximo de filamentos branquiales (20), la distribución de las branquias (hasta el setígero 115) y la fórmula maxilar, principalmente de la maxila IV $(8+10,7+9)$. E. pennata ha sido registrada principalmente en la región Antártica y Ártica. Otras dos especies muy semejantes morfológicamente, $E$. antarctica Baird y E. narconi Baird, han sido consideradas por Orensanz (1990), como sinónimas de E. pennata.

Distribución geográfica: sector Atlántico del Ártico; Atlántico norte desde el estrecho Davis y Newfoundland a frente a la Florida, y desde Spitzbergen al Mediterráneo; sector Atlántico de la Antártica (islas Georgias, Orcadas y Shetland del Sur, península Antártica, paso Drake); Atlántico sur, alrededor de África del Sur y desde las islas Falkland hasta la Provincia de Buenos Aires (Orensanz, 1975); Chile, desde la región de Aysén (este estudio) hasta frente al Campo de Hielo Patagónico Sur (Montiel et al., 2004).

\section{ONUPHIDAE Kinberg Onuphis pseudoiridescens Averincev, 1972} (Figs. 3i-3o)

Onuphis (Nothria) pseudoiridescens Averincev, 1972: 176, pl. 32, figs. 1-9. (Not Johnson, 1901).

Onuphis pseudoiridescens: Orensanz, 1976: 36; 1990: 20-23, pl. 1; Rozbaczylo, 1985: 111.

Onuphis iridescens: Monro, 1930: 132-133; 1936: 150-151.

Nothria iridescens in Wesenberg-Lund, 1962: 109; Hartman, 1964: 113, pl. 35, figs. 7-8; HartmannSchröder, 1965: 159-161, figs. 129-131.

Material examinado: Cimar-7: E-6 (18 ejemplares, SSUC 7187), E-41 (2 ejemplares, SSUC 7188); Cimar-8: E-1 (2 ejemplares, MNHNCL-An 2039), E-6 (2 ejemplares, MNHNCL-An 2040), E-8 (1 ejemplar, 


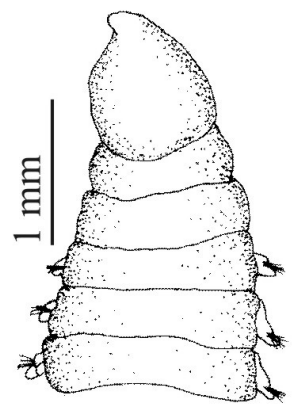

a
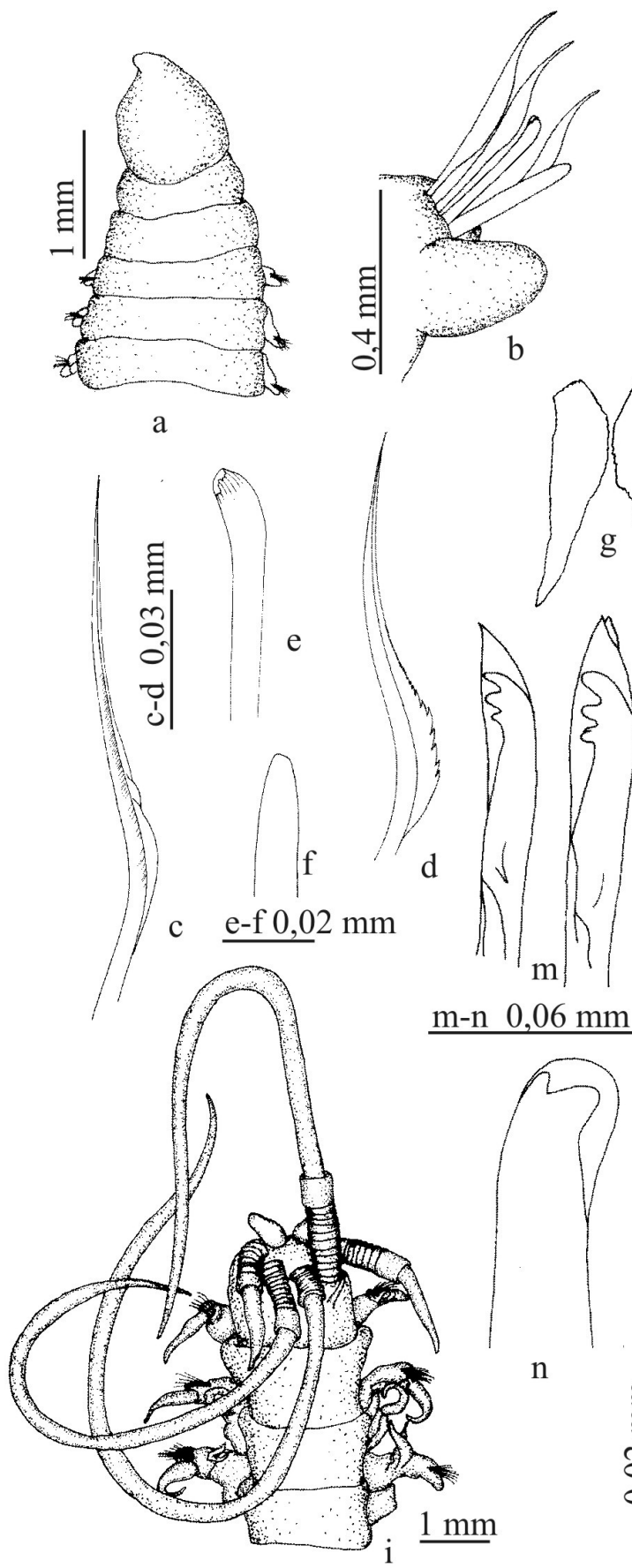
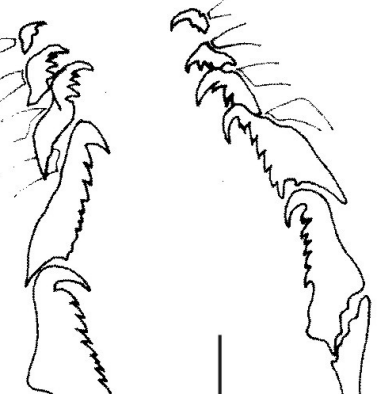

छే

$\mathrm{h}$
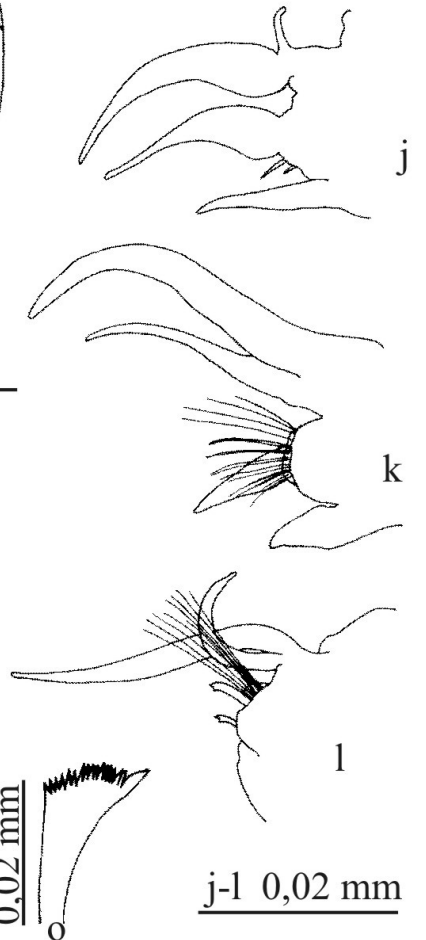

Figura 3. Notocirrus cf. virginis: a) extremo anterior, en vista dorsal, b) parápodo medio-anterior, c) seta limbada con el margen liso, d) seta limbada con el margen serrado, e) seta acicular, f) acícula, g) mandíbulas, h) complejo maxilar; Onuphis pseudoiridescens: i) extremo anterior, en vista dorsal, j) primer parápodo, k) tercer parápodo, l) parápodo posterior, $m$ ) ganchos seudocompuestos, $n$ ) seta acicular, o) seta pectinada.

Figure 3. Notocirrus cf. virginis: a) anterior end in dorsal view, b) middle anterior parapodium, c) winged seta with smooth edge, d) winged seta with serrated edge, e) acicular seta, f) aciculum, g) mandibles, h) jaws; Onuphis pseudoiridescens: i) anterior end in dorsal view, j) first parapodium, k) third parapodium, l) posterior parapodium, m) pseudocompound hooks, n) acicular seta, o) comb seta. 
SSUC 7189), E-51 (9 ejemplares, SSUC 7190), E53 (8 ejemplares, SSUC 7191), E-76 (2 ejemplares, SSUC 7192).

Caracterización: el ejemplar de mayor tamaño, con 97 setígeros, midió $52 \mathrm{~mm}$ de largo y $4,5 \mathrm{~mm}$ de ancho. Prostomio con un par de antenas globulares; tentáculos occipitales con cirróforos largos con 10-12 anillos (Fig. 3i); el tentáculo medio, de mayor longitud, se extiende hasta el setígero 8 y es $31 \frac{1}{2}$ veces más largo que los más externos. Ojos ausentes. Los cirros peristomiales son filiformes y se extienden hasta el margen posterior del prostomio. Las branquias son cirriformes desde el primer setígero (Fig. 3j) y se continúan hasta la región media-posterior del cuerpo. Parápodos birremos; cirro dorsal de los dos primeros setígeros de igual longitud que el lóbulo parapodial; a partir del tercer setígero (Fig. 3k) el lóbulo parapodial comienza a acortarse hasta convertirse, en los setígeros posteriores, en un lóbulo redondeado (Fig. 31). Setas capilares con margen finamente aserrado; ganchos encapuchados pseudocompuestos (Fig. 3mn) distalmente tridentados en los primeros cuatro setígeros, en algunos el diente proximal puede ser bífido por lo cual el gancho se observa tetradentado. Setas pectinadas con 15-17 dientes, siendo el más externo de mayor longitud (Fig. 3o). Gancho subacicular encapuchado bidentado, presente a partir del setígero 12-15. Aparato maxilar con la maxila I falcada; $\mathrm{II}=$ 7+7; III = 8+0; IV = 8+7; V = 1+1.

Observaciones: Orensanz (1990), señala que las branquias de esta especie aparecen desde el primer setígero, pero que en algunos casos pueden aparecer después del cuarto setígero. En los ejemplares examinados por nosotros, sólo dos presentaron branquias a partir de segmentos posteriores al primer setígero (2 y 4). Orensanz (1990), también señala que esta especie es muy semejante a $O$. iridescens (Johnson) y O. opalina (Verrill); de la primera se diferencia por la forma aguzada de las valvas de los ganchos tridentados, mientras que con la segunda se diferencia en la distribución geográfica, estableciendo que son especies disjuntas: la primera restringida a aguas templadas de América del Sur y la segunda a aguas templadas del Atlántico noroccidental. Fauchald (1982), describe dos especies nuevas, O. heterodentata y $O$. lithobiformis, a partir del registro de Nothria iridescens de Hartman (1967); sin embargo, Orensanz (1990) considera que ellas son sinónimas de $O$. pseudoiridescens.

Distribución geográfica: alrededor del sur de Sudamérica; frente a la Patagonia Argentina; alrededor de las Islas Falkland (Orensanz, 1990); Chile, desde Punta Galera (Hartmann-Schröder, 1965) al Estrecho de Magallanes (Gambi \& Mariani, 1999).

\section{CLADO SPIONIDA}

\section{CHAETOPTERIDAE Malmgren}

Chaetopterus cf. variopedatus (Renier, 1804)

(Figs. 4a-4c)

Tricoelia variopedata Renier, 1804: 18.

Chaetopteus variopedatus: Ehlers, 1897: 109-110; 1900:219; Fauvel 1927: 77-79, fig. 26 a-n; Hartman, 1953: 45; 1966: 25, pl. 6, figs. 7-9; Day, 1967: 529, fig. 22.2.a-g; Kohn \& Lloyd, 1973: 702; Hutchings \& Rainer, 1979: 774-775; Gilbert, 1984: 11/5-6, fig. 11.2a-e; Hutchings \& Murray, 1984: 63; HartmannSchröder, 1985: 83; Rozbaczylo, 1985: 149.

Chaetopterus antarcticus Kinberg, 1867: 338.

Material examinado: Cimar-7: E-29 (1 ejemplar, SSUC 7159). Cimar-8: E-53 (1 ejemplar, SSUC 7160).

Caracterización: los dos ejemplares están incompletos y muy deteriorados. Región cefálica con dos palpos cortos (Fig. 4a). Notosetas de la región anterior lanceoladas (Fig. 4b). Cuarto setígero con una seta modificada (Fig. 4c). Uncinos pectinados con 8-10 dientes.

Observaciones: los ejemplares fueron determinados como Ch. cf. variopedatus por la presencia de un par de palpos cortos en la región anterior, las setas lanceoladas y las características de las setas del cuarto setígero. Sin embargo, debido al mal estado de los ejemplares no fue posible realizar observaciones detalladas de los uncinos, setas capilares y morfología de los segmentos medios y posteriores. Day (1967) y Gilbert (1984) señalan hasta 6 setas modificadas, sin embargo, en nuestros ejemplares solo se observó una sola seta de este tipo. Petersen (1984) indica que la especie Chaetopterus variopedatus es un complejo de especies y que el género corrientemente ha sido considerado monotípico con una especie cosmopolita altamente variable, $C h$. variopedatus, originalmente descrita para el mar Adriático. En este sentido, posiblemente nuestra especie podría ser asignada a $C h$. antarcticus descrita por Kinberg en 1867 para el Estrecho de Magallanes, hasta ahora considerada como sinónimo de Ch. variopedatus.

Distribución geográfica: cosmopolita; Ártico; Antártica (Day, 1967); en Chile, desde Aysén (este estudio) a Isla Hermite (Monro, 1930); isla de Pascua (Kohn \& Lloyd, 1973). 


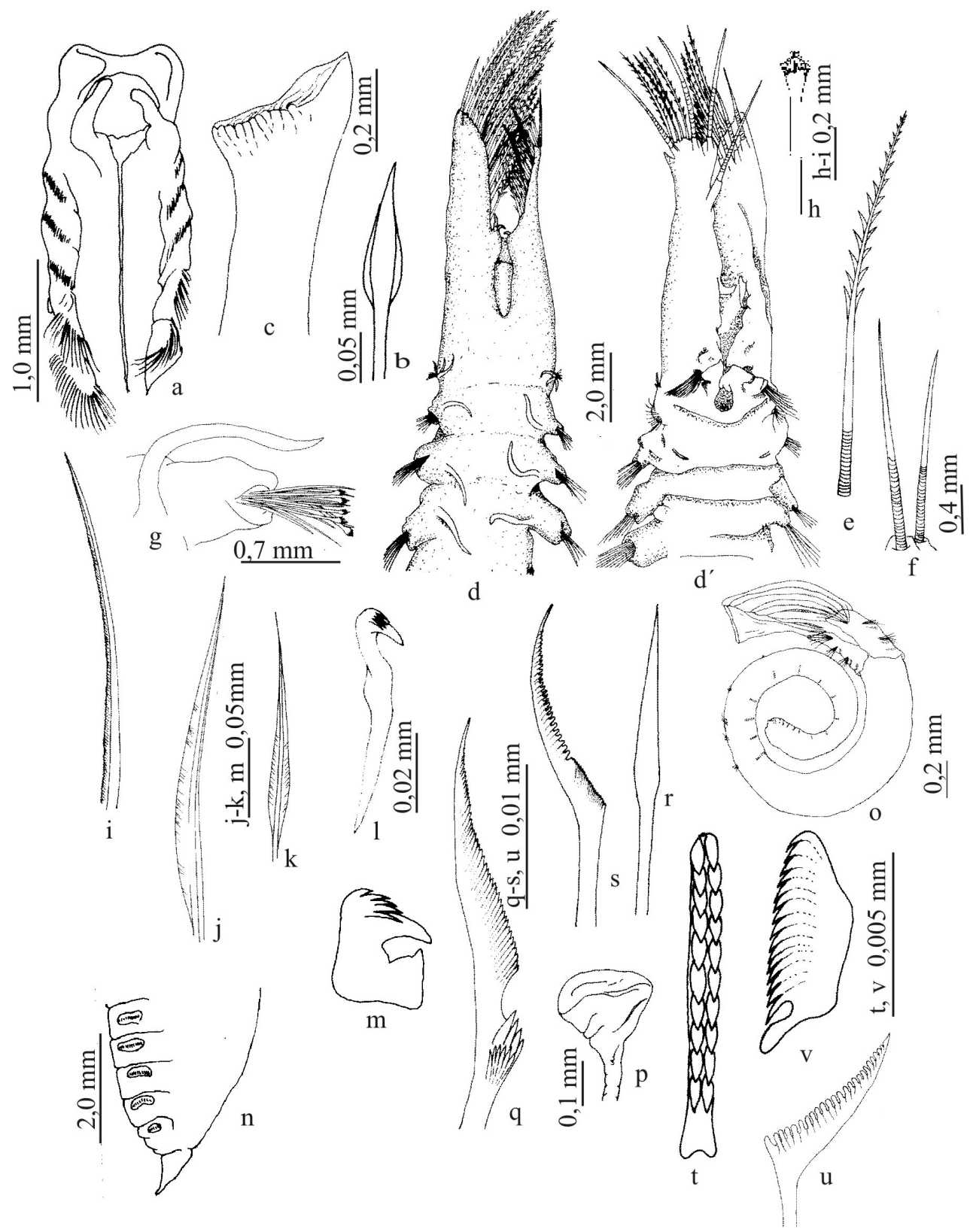

Figura 4. Chaetopteus cf. variopedatus: a) región anterior, en vista ventral, b) notoseta lanceolada, c) seta modificada; Idanthyrsus armatus: d) extremo anterior, en vista dorsal, d') extremo anterior, en vista ventral, e) seta opercular con procesos espiniformes, f) setas operculares internas, g) parápodo de un segmento paratorácico, h) palea torácica, i) seta capilar; Fabricinae indeterminado: j) notoseta limbada, k) palea subespatulada, l) neuroseta torácica, m) uncino abdominal, n) extremo posterior en vista latero-ventral; Paralaeospira levinseri: o) vista general de un ejemplar completo, p) opérculo, q) seta del collar, r) seta simple, s) seta segadora, t) uncino torácico, u) seta geniculada, v) uncino abdominal.

Figure 4. Chaetopteus cf. variopedatus: a) anterior end in ventral view, b) notoseta from anterior region, c) enlarged seta of setiger 4; Idanthyrsus armatus: d) anterior end in dorsal view, d') anterior end in ventral view, e) opercular seta with spiniform processes, f) inner opercular setae, g) parapodium of a parathoracic segment, h) thoracic palea, i) capillary seta; Fabricinae indeterminado: j) winged notoseta, k) subspatulate palea, l) thoracic neuroseta, m) abdominal uncinus, n) posterior end in ventrolateral view; Paralaeospira levinseri: o) general view of a complete specimen, p) operculum, q) collar seta, r) simple seta, s) sickle seta, t) thoracic uncinus, u) abdominal seta, v) abdominal uncinus. 


\section{CLADO SABELLIDA}

\section{SABELLARIIDAE Johnston Idanthyrsus armatus Kinberg, 1867}

(Figs. 4d-4i)

Idanthyrsus armatus Kinberg, 1867: 350; Monro, 1930: 177, fig. 73; Hartman, 1944b: 336-337, pl. 31, fig. 36; Hartmann-Schröder, 1979: 149-150; Carrasco \& Bustos, 1981: 170-172, figs. 8-11; Lana \& Bremec, 1994: 210-211.

Material examinado: Cimar-8: E-78 (1 ejemplar, MNHNCL-An 2037), E-63 (2 ejemplares, SSUC 7161).

Caracterización: un ejemplar de $15 \mathrm{~mm}$ de longitud midió 1,9 mm de ancho. Opérculo con dos filas de paleas color ámbar (Fig. 4d-d'); las más externas son rectas con procesos espiniformes (Fig. 4e), y las más internas son robustas con estriaciones transversales (Fig. 4f). Dos pares de ganchos nucales. Con un haz de setas capilares a ambos lados de la cavidad bucal. Los primeros dos setígeros con setas bilimbadas cortas. Con tres segmentos paratorácicos (Fig. 4g) que llevan aproximadamente cinco pares de paleas por segmento, con el extremo distal hirsuto (Fig. 4h), acompañadas de setas capilares con margen aserrado (Fig. 4i). Branquias filiformes, el primer notópodo con tres filamentos, y los siguientes con un filamento. Uncinos abdominales con dos filas de siete dientes.

Observaciones: los ejemplares examinados concuerdan bien con las características señaladas para la especie, sin embargo, Monro (1930), Hartman (1944b), Carrasco \& Bustos (1981) y Lana \& Bremec (1994) no mencionan el haz de setas capilares a ambos lados de la cavidad bucal, así como tampoco las notosetas bilimbadas cortas en los primeros setígeros, ni las branquias.

Distribución geográfica: Sudamérica; costa del Pacífico de Colombia; Islas Falkland; Isla South Georgia; plataforma continental frente a la Patagonia y norte de Argentina; México occidental; Columbia Británica; Alaska; Australia; Japón (Lana \& Bremec, 1994); en Chile, de Valparaíso (Kinberg, 1867) al Canal Beagle (Ehlers, 1897).

\section{SABELLIDAE Malmgren Fabricinae indeterminado} (Figs. 4j-4n)

Material examinado: Cimar-8: E-6 (1 ejemplar, SSUC 7162).

Caracterización: ejemplar incompleto, sin la corona radial; collar bien desarrollado, con una escotadura dorsal, márgenes laterales lisos y ventralmente con dos pequeñas elevaciones. Notosetas torácicas bilimbadas (Fig. 4j) y páleas subespatuladas (Fig. 4k). Neurosetas torácicas sin setas acompañantes y con manubrio largo (Fig. 41), neurosetas abdominales con base cuadrada (Fig. $4 \mathrm{~m}$ ). Pigidio con un pequeño apéndice caudal (Fig. 4n).

Observaciones: las características de las setas, así como la ausencia de setas acompañantes en los uncinos torácicos, nos permiten ubicar el ejemplar en la subfamilia Fabricinae y posiblemente en el género Chone Kröyer, por la forma del uncino abdominal. El ejemplar examinado difiere de las dos especies de Chone registradas en la costa de Chile: Chone rosea Hartmann-Schröder, 1965 y Chone striata HartmannSchröder, 1965, porque presenta páleas subespatuladas, además presenta un pequeño apéndice caudal en el pigidio, mientras que en $C h$. rosea y Ch. striata el extremo distal es redondeado. Respecto a la forma del extremo distal, dos especies también presentan apéndices caudales: $C h$. filicaudata Southern y $C h$. americana Day, pero ambas también presentan páleas espatuladas.

\section{SERPULIDAE Savigny Apomatus sp.}

(Figs. 5a-5h)

Material examinado: Cimar 8: E-59 (3 ejemplares, SSUC 7163), E-71 (1 ejemplar, SSUC 7164).

Caracterización: sólo dos ejemplares están completos; el de mayor tamaño mide $26,5 \mathrm{~mm}$ de largo y 3,4 mm de ancho. Región torácica con siete setígeros y la abdominal con 118. Con quince pares de radiolas. Opérculo (Fig. 5a) esférico, membranoso, sobre el extremo distal de la segunda radiola dorsal; pseudopérculo presente (Fig. 5b). Setas del collar bilimbadas (Fig. 5c). Notosetas torácicas bilimbadas y tipo "Apomatus" (Fig. 5d-d'). Uncinos torácicos, desde el segundo setígero, con dos filas de 24-28 dientes (Fig. 5e), y el extremo basal con una pequeña escotadura, pero sin llegar a ser bidentado. Notosetas abdominales geniculadas (Fig. $5 \mathrm{f}-\mathrm{f}^{\prime}$ ) con el borde cortante liso; en los segmentos posteriores éstas son reemplazadas por largas y delgadas setas capilares. Uncinos abdominales con dos filas de 12-18 dientes (Fig. 5 g). Pigidio con dos pequeñas papilas (Fig. $5 \mathrm{~h}$ ). Tubo blanco frágil, sin anillaciones ni crestas.

Observaciones: Ten Hove \& Wolf (1984) se refieren al confuso estado taxonómico del complejo Apomatus señalado por Zibrowius (1968). Tal confusión se 


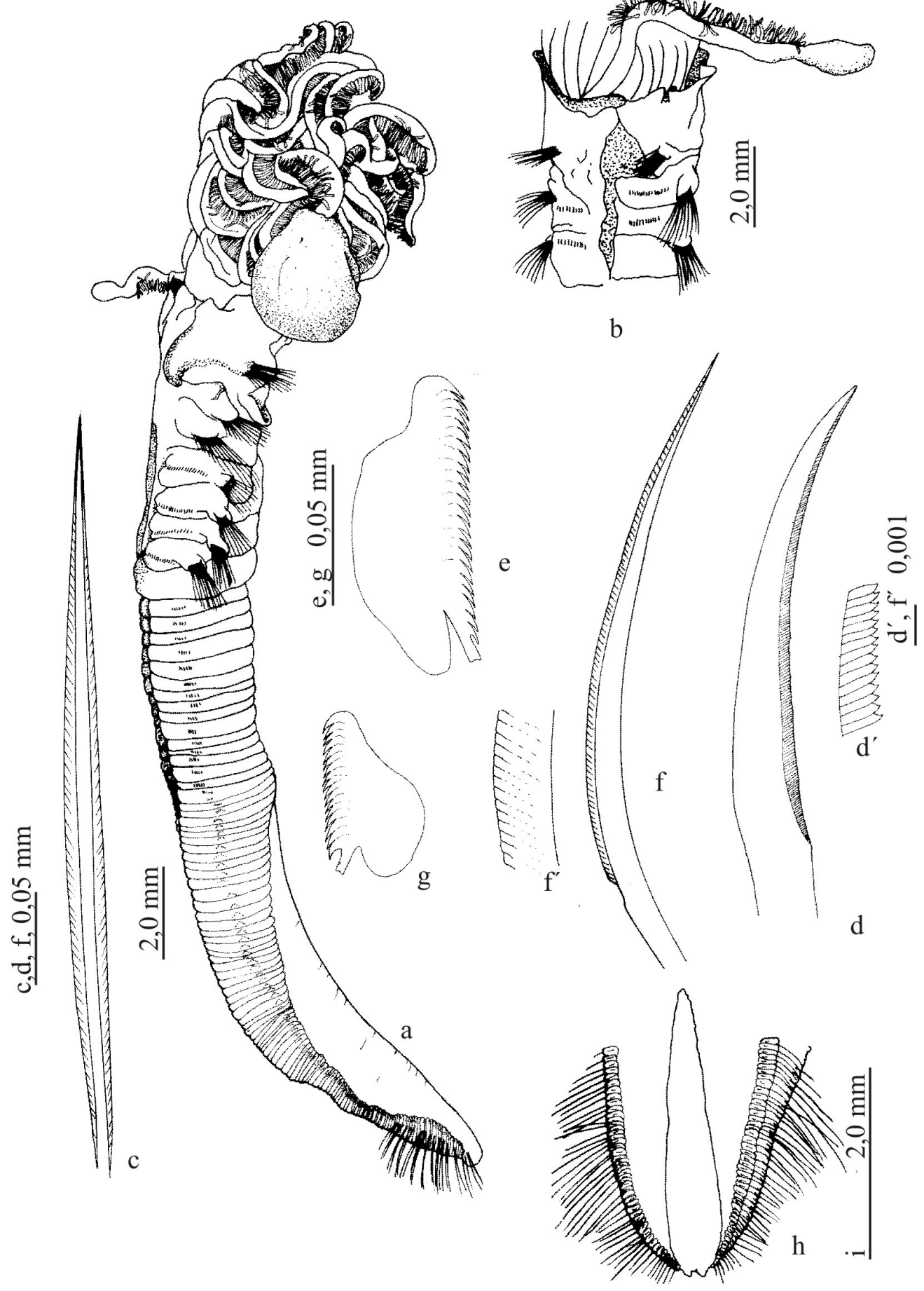

Figura 5. Apomatus sp.: a) vista general del ejemplar, b) porción del extremo anterior mostrando el seudopérculo, c) seta del collar, d-d') seta tipo "Apomatus", e) uncino torácico, f-f') notoseta abdominal geniculada, g) uncino abdominal, h) extremo posterior en vista ventral.

Figure 5. Apomatus sp.: a) general view of the specimen, b) anterior end showing the pseudoperculum, c) collar seta, d-d') seta of Apomatus, e) thoracic uncinus, f-f') abdominal notoseta, g) abdominal uncinus, h) posterior end in ventral view. 
basa en la designación de setas tipo "Apomatus" en especies operculadas y no operculadas, señalando, en este último caso, que el opérculo de tales especies se había desprendido y que ello podría explicar la contradicción entre ambos grupos de serpúlidos con este tipo particular de setas. Sin embargo, Ten Hove \& Wolf (1984), plantean la necesidad de realizar una revisión detallada de las especies referidas a este género que carecen de opérculo y presentan la mencionada seta. Por su parte, Kupriyanova \& Jirkov (1997) señalan que las especies del género Apomatus deben considerarse como sinónimas de Protula. En comparación con otras especies operculadas, pertenecientes al género, los ejemplares examinados aquí se diferencian de A. brownii Pixell, registrada para la Antártica, por el mayor número de setas del collar (> 12 setas), en vez de las 5-7 en A. brownii. Por otro lado, Pixell (1913: 358, Fig. 2a), señala que las setas del collar presentan un pequeño talón aserrado y que el extremo distal de la misma es igualmente aserrado. Se diferencia de A. similis Marion \& Bobretzky, porque ésta presenta setas con forma de hoz y 4-18 manchas oculares en los ejes de las radiolas; de $A$. lille $i$ Benham, porque las notosetas torácicas, además de las del tipo "Apomatus", presenta unilimbadas y no bilimbadas. Benham (1927), hace referencia a un pequeño haz de setas limbadas en el collar dirigidas hacia adelante. También se diferencia de A. ampulliferus Phillippi por la presencia de 10-15 manchas oculares en el eje de la radiola así como en el collar.

\section{Paralaeospira levinseni (Caullery \& Mesnil, 1897)} (Figs. 4o-4v)

Spirorbis (Paralaeospira) levinseni Caullery \& Mesnil, 1897: 207, pl. 8, fig. 14.

Paralaeospira levinseni: Knight-Jones \& Walker, 1972: 33-35, fig.1; Vine, 1977: 17-20; fig. 8; Rozbaczylo, 1985: 219.

Material examinado: Cimar-8: E-67 (5 ejemplares, SSUC 7165).

Caracterización: el tubo es sinestroso, liso. El ejemplar de mayor tamaño mide 2,5 mm de longitud y 0,2 mm de ancho (Fig. 4o). Opérculo ovalado, plano o ligeramente cóncavo (Fig. 4p). Collar torácico no fusionado, con cuatro setígeros, tres uncinígeros. Setas del collar con talón fuertemente dentado y hoja finamente dentada (Fig. 4q) y setas capilares asociadas. Segundo y tercer setígero con seta simple (Fig. 4r) y seta segadora (Fig. 4s). Uncinos torácicos con 2 ó 3 filas longitudinales de 11-12 dientes y extremo basal ligeramente ensanchado. (Fig. 4t). Región abdominal con 16 segmentos; seta geniculada (Fig. $4 u$ ) con un prominente talón y 20 dientes redondeados. Uncinos abdominales con aproximadamente 10 filas longitudinales de 10-12 dientes (Fig. 4v).

Observaciones: Knight-Jones \& Walker (1972) y Vine (1977) señalan que en la superficie del opérculo de algunos ejemplares adultos se observa una pequeña estructura en forma de gancho y una pequeña papila transparente, sólo visibles al aclarar el opérculo. Esta especie incuba sus huevos en el interior del tubo, adosados al cuerpo, dispuestos en dos hileras de 7-9 huevos; esto sólo se observó en un ejemplar.

Distribución geográfica: cabo Denison, Antártica; Islas Orcadas del Sur; Ciudad del Cabo, Sudáfrica (Vine, 1977); en Chile, desde Talcahuano, Bahía de Concepción (Knight-Jones \& Walker, 1972) a Bahía Orange, Patagonia (Caullery \& Mesnil, 1897).

\section{CLADO SCOLECIDA}

MALDANIDAE Malmgren Asychis amphiglypta (Ehlers, 1897)

(Figs. 6a-6f)

Maldane amphiglypta Ehlers, 1897: 119-122, pl. 8, figs. 187-193.

Asychis amphiglypta: Arwidsson, 1911: 35-40, pl. 1, figs. 27-31, pl. 2, figs. 55-58; Hartman, 1966: 59, 61, pl. 19, fig. 6; 1967: 144; Hartmann-Schröder \& Rosenfeltd, 1989: 79; Rozbaczylo, 1985: 175; Hartmann-Schröder, 1986: 86.

Material examinado: Cimar-8: E-76 (1 ejemplar, SSUC 7166).

Caracterización: el ejemplar completo, con 14 setígeros, mide $56,5 \mathrm{~mm}$ de largo y $2,1 \mathrm{~mm}$ de ancho. Placa cefálica oblicua (Fig. 6a), con márgenes lisos divididos en tres lóbulos por un par de hendiduras laterales. Primer setígero sin neurosetas. Setas bilimbadas (Fig. 6b) y capilares con constricciones (Fig. 6c). Ganchos rostrados con tres filas de dentículos sobre el diente principal y cinco barbillones subdistales (Fig. 6d). Segmento preanal asetígero. Placa anal sin cirros, con dos pequeñas escotaduras laterales (Fig. 6e-f). Ano dorsal.

Observaciones: el ejemplar examinado concuerda bien con la descripción de Ehlers (1897), y parcialmente con las de Hartman (1966) y HartmannSchröder \& Rosenfeltd (1989) quienes señalan la presencia de hasta tres cirros anales. 

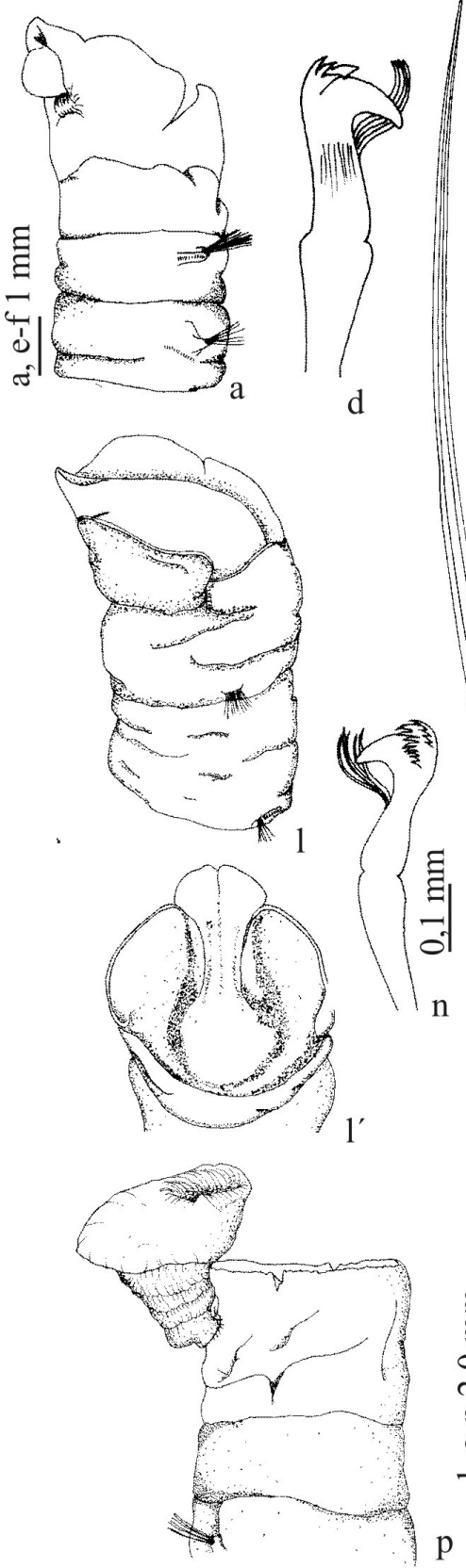

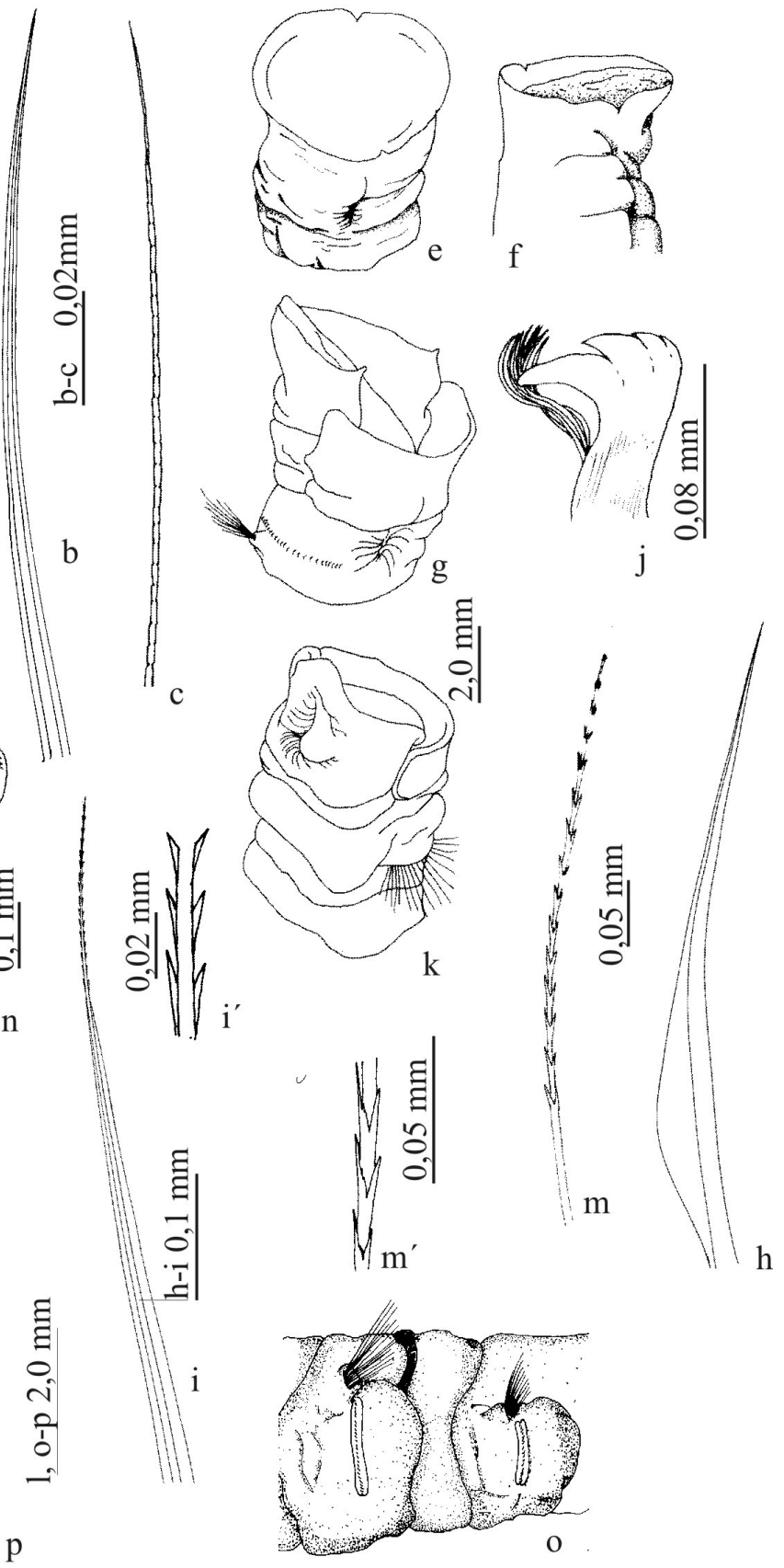

Figura 6. Asychis amphiglypta: a) extremo anterior, en vista lateral, b) seta bilimbada, c) seta capilar, d) gancho rostrado, e-f) placa anal; Maldane chilensis: g) placa cefálica, h) seta limbada, i) seta bilimbada, i') detalle del extremo de la cerda bilimbada, j) gancho rostrado $k$ ) placa anal; Maldane sarsi antarctica: I) extremo anterior en vista lateral, l') extremo anterior en vista dorsal, $m$-m') seta espinulosa, n) gancho rostrado, o) detalle de la cresta glandular del quinto setígero, p) placa anal.

Figure 6. Asychis amphiglypta: a) anterior end in lateral view, b) winged capillary, c) capillary seta, d) normal hook, e-f) anal plate; Maldane chilensis: g) cephalic plate, $h$ ) winged seta, i) winged capillary, $i$ ') detail of the distal end of a winged capillary, $\mathbf{j}$ ) normal hook, $\mathrm{k}$ ) anal plate; Maldane sarsi antarctica: $\mathrm{l}$ ) anterior end in lateral view, l') anterior end in dorsal view, m-m') feathered capillary, n) normal hook, o) detail of the glandular crest in the fifth setiger, p) anal plate. 
Distribución geográfica: Nueva Zelanda; Islas Falkland; Isla South Georgia; Antártica (HartmannSchröder \& Rosenfeldt, 1989); en Chile, desde Aysén (este estudio) al Estrecho de Magallanes (Gambi \& Mariani, 1999).

\section{Maldane chilensis Hartmann-Schröder, 1965}

(Figs. 6g-6k)

Maldane chilensis Hartmann-Schröder, 1965: 239242, figs. 238-241; Rozbaczylo, 1985: 177.

Material examinado: Cimar-7: E-6 (1 ejemplar, SSUC 7150), E-25 (1 ejemplar, SSUC 7149), E-32 (1 ejemplar, SSUC 7151). Cimar-8: E-49 (4 ejemplares, SSUC 7152), E-51 (42 ejemplares, SSUC 7148; 3 ejemplares, MNHNCL-An 2038), E-53 (4 ejemplares, SSUC 7153).

Caracterización: el ejemplar de mayor tamaño, con 19 setígeros, mide $93 \mathrm{~mm}$ de longitud y $6,2 \mathrm{~mm}$ de ancho. Placa cefálica bilobulada (Fig. 6g); el lóbulo dorsal con tres pequeñas proyecciones, el ventral con margen liso. Setas limbadas (Fig. 6h) y bilimbadas distalmente espinulosas (Fig. 6i-i'). Ganchos rostrados con tres crestas concéntricas de dentículos sobre un diente principal y 7-9 barbillones subdistales (Fig. 6j). Dos segmentos preanales asetígeros. Placa anal plana, con dos escotaduras profundas y márgenes lisos (Fig. 6k). Ano dorsal.

Distribución geográfica: hasta ahora conocida sólo en Chile, de Valparaíso (Hartmann-Schröder, 1965) a Aysén (este estudio).

\section{Maldane sarsi antarctica Arwidsson, 1911}

(Figs. 61-6p)

Maldane sarsi antarctica Arwidsson, 1911: 32-35, pl. 1, figs. 23-26, pl. 2, figs. 50-54; Monro, 1930: 169; Hartman, 1966: 66, pl. 21, figs. 10-11; Rozbaczylo, 1985: 177-178; Hartmann-Schröder, 1986: 86-87; Hartmann-Schröder \& Rosenfeldt, 1989: 79; Knox \& Cameron, 1998: 77-78, figs. 149-150.

Material examinado: Cimar-7: E-6 (1 ejemplar, SSUC 7154), E-21 (1 ejemplar, SSUC 7155), E21A (1 ejemplar, SSUC 7156), E-22 (93 ejemplares, SSUC 7157), E-41 (34 ejemplares, SSUC 7158).

Caracterización: el ejemplar de mayor tamaño mide $123,5 \mathrm{~mm}$ de largo y $3,7 \mathrm{~mm}$ de ancho, con 19 segmentos torácicos y dos segmentos preanales asetígeros. Placa cefálica con el margen liso y dos escotaduras laterales y una cresta fuertemente elevada y arqueada (Fig. 61-1'), surcos nucales cortos. Primer setígero sólo con notosetas capilares limbadas; en los setígeros siguientes, en adición a las capilares hay setas espinulosas (Fig. 6m-m'). Con ganchos rostrados a partir del segundo setígero (Fig. 6n), con una arco de 10-12 dentículos sobre el diente principal y una cresta de dentículos diminutos. Sobre la superficie dorsal del quinto setígero se observa una delgada cresta glandular (Fig. 6o). Placa anal débilmente crenulada, con dos pequeñas incisiones laterales (Fig. 6p).

Observaciones: los ejemplares examinados concuerdan bien con las características señaladas por Arwidsson (1911) y Hartman (1966). Esta subespecie es muy semejante a $M$. sarsi Malmgren, de la cual se diferencia porque esta última carece de la banda glandular dorsal sobre el quinto setígero y porque la cresta cefálica no es tan elevada.

Distribución geográfica: estrecho Bransfield; costa de Graham; Isla South Georgia; Islas Orcadas del Sur; Mar de Ross (Knox \& Cameron, 1998); Australia (Hartmann-Schröder, 1986); en Chile, desde la región de Aysén (este estudio) hasta el Estrecho de Magallanes (Gambi \& Mariani, 1999).

\section{DISCUSIÓN}

El conocimiento general sobre la riqueza de especies de poliquetos bentónicos de la región de Aysén, proviene principalmente de las publicaciones de Wesenberg-Lund (1962), Hartmann-Schröder (1962, 1965), Cañete et al. (1999), Rozbaczylo et al. (2005) y Rozbaczylo et al. (2006), con un total de 77 especies registradas entre las zonas intermareal y submareal ( $450 \mathrm{~m}$ de profundidad). Con la información presentada aquí aumentan a 91 las especies de poliquetos bentónicos submareales de la región de Aysén, producto de la incorporación de catorce nuevos registros de especies de los clados Amphinomida, Eunicida, Spionida, Sabellida y Scolecida. Tres pertenecen a la familia Maldanidae (A. amphiglypta, M. chilensis y M. sarsi antarctica), dos a las familias Lumbrineridae (E. chilensis y $N$. leptognatha) y Serpulidae (P. levinseni y Apomatus sp.) y una a Amphinomidae (P. chilensis), Oenonidae (N. cf. virginis), Eunicidae (E. pennata), Onuphidae (O. pseudoiridescens), Chaetopteridae (Ch. cf. variopedatus), Sabellariidae (I. armatus) y Sabellidae (Fabricinae indeterminado), respectivamente.

Los muestreos efectuados en los cruceros Cimar7 y Cimar-8 Fiordos, realizados en la zona bentónica a profundidades $\sim 450 \mathrm{~m}$, han permitido ampliar el rango de distribución batimétrica de Schistomeringos 
longicornis hasta los $110 \mathrm{~m}$, la que habitualmente había estado restringida a la zona submareal somera (<30 m de profundidad) (Cañete et al., 1999) y de las especies A. amphiglypta, M. chilensis, $M$. sarsi antarctica, P. levinseni, Apomatus sp., Ch. cf. variopedatus, I. armatus y Fabricinae indeterminado entre los 30 y $304 \mathrm{~m}$ de profundidad.

Los patrones de distribución geográfica de los taxa de los clados Amphinomida, Eunicida, Spionida, Sabellida y Scolecida permiten definir a las especies $S$. longicornis, $N$. leptognatha, E. pennata, $N$. cf. virginis y $O$. pseudoiridescens, $C h$. cf. variopedatus, Fabricinae indeterminado, A. amphiglypta, Apomatus sp., M. chilensis y $M$. sarsi antarctica, como restringidas a aguas templadas frías dentro de la Provincia Magallánica, alcanzando en general como límite más septentrional de distribución la zona de Chiloé (ca. $\left.41^{\circ} \mathrm{S}\right)$. Por otro lado, las especies P. chilensis, E. chilensis, I. armatus y P. levinseni presentan una amplia distribución a lo largo de la costa de Chile, abarcando principalmente desde aguas templadas cálidas ( $c a$. $29^{\circ} \mathrm{S}$ ) de la Provincia Peruana hasta templadas frías (ca. 55 $\mathrm{S}$ ) de la Provincia Magallánica (Hernández et al., 2005; Moreno et al., 2006).

Los cruceros Cimar-7 y Cimar-8 Fiordos han permitido aumentar el conocimiento de la biodiversidad marina, en términos de ampliación de rangos geográficos y batimétricos de poliquetos bentónicos submareales de fondos blandos de la región de Aysén, tanto de los clados Phyllodocida (Rozbaczylo et al., 2005) y Terebellida (Rozbaczylo et al., 2006), como Amphinomida, Eunicida, Spionida, Sabellida y Scolecida (este trabajo).

\section{AGRADECIMIENTOS}

Los autores agradecen al Comité Oceanográfico Nacional por el apoyo logístico prestado durante la realización de los cruceros Cimar-7 y Cimar-8 Fiordos. También agradecemos a Patricio Zavala por la colaboración técnica en la catalogación y posterior ingreso de los taxa de poliquetos bentónicos a la sala "Colección de Flora y Fauna Profesor Patricio Sánchez Reyes" de la Pontificia Universidad Católica de Chile y en el Museo Nacional de Historia Natural. Hacemos extensivos nuestros agradecimientos a tres revisores anónimos por sus valiosas sugerencias y comentarios críticos. Este trabajo fue realizado como parte del proyecto "Estudio sobre poliquetos marinos bentónicos de los canales occidentales de la XI Región y del océano Pacífico adyacente", financiado por el Servicio Hidrográfico de la Armada de Chile, en el marco del Crucero Cimar 8-Fiordos.

\section{REFERENCIAS}

Arwidsson, I. 1911. Die Maldaniden. Wissenschaftliche Ergebnisse der Schwedischen Südpolar-Expedition 1901-1903, 6(6): 1-44.

Averincev, V.G. 1972. [Benthic polychaetes Errantia from the Antarctic and Subantarctic collected by the Sovietic Antarctic Expeditions]. (En Ruso). Biol. Res. Sovietic Antarctic Expeditions, 5: 88-293.

Benham, W.B. 1927. Polychaeta. British Antarctic Terra Nova Expedition, 1910. Nat. Hist. Rep., Zool., 7(2): 47-182.

Cañete, J.I., G.L. Leighton \& F.F. Aguilera. 1999. Polychaetes from Aysén Fjord, Chile: distribution, abundance and biogeographical comparison with the shallow soft-bottom polychaete fauna from Antarctica and the Magellan Province. Sci. Mar., 63(1): 243-252.

Carrasco, F.D. \& H.E. Bustos. 1981. Dos especies de poliquetos de la familia Sabellariidae, Sabellaria minuta sp. n. e Idanthyrsus armatus Kinberg, epizoos de crustáceos decápodos. Bol. Soc. Biol. Concepción, 51: 167-173.

Caullery, M. \& F. Mesnil. 1897. Etudes sur la morphologie comparée et la phylogénie des espèces chez les Spirorbes. Bull. Scient. Fr. Belg., 30: 185-233.

Day, J.H. 1967. A monograph on the Polychaeta of Southern Africa. Brit. Mus. Nat. Hist. Publ., 656: $1-878$.

Ehlers, E. 1897. Polychaeten. Hamburger Magalhaenischen Sammelreise. Hamburg, 148 pp.

Ehlers, E. 1900. Magellanische Anneliden gesammelt während der schwedischen Expedition nach den Magellansländern. Nachr. K. Ges. Wiss. Göttingen, 1900: 206-223.

Ehlers, E. 1901. Die Polychaeten des magellanischen und chilenischen Strandes. Ein faunistischer Versuch. Festschrift zur Feier des Hundertfünfzigjährigen Bestehens der Königlichen Gesellschaft der Wissenschaften zu Göttingen. (Abh. Math.-Phys. K.), Berlin, 232 pp. 
Fauchald, K. 1977. The polychaete worms: definitions and keys to the orders, families and genera. Nat. Hist. Mus. Los Angeles County, Sci. Ser., 28: 1-190.

Fauchald, K. 1982. Some species of Onuphis (Polychaeta: Onuphidae) from the Atlantic Ocean. Proc. Biol. Soc. Wash., 95(2): 238-250.

Fauchald, K. 1992. A review of the genus Eunice (Polychaeta: Eunicidae) based upon type material. Smithsonian Contrib. Zool., 523:1-422.

Fauvel, P. 1927. Polychètes sédentaires. Addenda aux errantes, Archiannélides, Myzostomaires. Faune de France, 16: 1-494.

Fauvel, P. 1936. Polychètes Expédition antarctique Belge. Résultats du voyage de la Belgica en 18971899 , sous le commandement de A. de Gerlache de Gomery, 46 pp.

Gambi, M.C. \& S. Mariani. 1999. Polychaetes of the soft bottoms of the Straits of Magellan collected during the Italian oceanographic cruise in FebruaryMarch 1991. Sci. Mar., 63(1): 233-242.

Gilbert, K.M. 1984. Family Chaetopteridae Malmgren, 1867. En: J.M. Uebelacker \& P.G. Johnson (eds.). Taxonomic guide to the polychaetes of the northern Gulf of Mexico. Vol. 2. Barry A. Vittor \& Associates, Mobile, Alabama. pp. 11.1-11.13

Glasby, K., P. Hutchings, K. Fauchald, H. Paxton, G. Rouse, C. Watson-Russel \& R. Wilson. 2000. Class Polychaeta. En: P.L. Beesley, G.J.B. Ross \& C.J. Glasby (eds.). Polychaetes \& Allies: The Southern Synthesis. Fauna of Australia. Vol. 4A. Polychaeta, Myzostomida, Pogonophora, Echiura, Sipuncula. CSIRO Publishing, Melbourne. Australia, pp. 1-296.

Hartman, O. 1944a. Polychaeteous annelids. V. Eunicea. Allan Hancock Pacific Exped., 10(1): 1-238.

Hartman, O. 1944b. Polychaetous annelids. VI. Paraonidae, Magelonidae, Longosomidae, Ctenodrilidae, and Sabellariidae. Allan Hancock Pacific Exped., 10(3): 311-389.

Hartman, O. 1948. The marine annelids erected by Kinberg with notes on some other types in the Swedish State Museum. Ark. Zool., 42A(1): 1137.
Hartman, O. 1953. Non-pelagic Polychaeta of the Swedish Antarctic Expedition 1901-1903. Further Zoological Results of the Swedish Antarctic Expedition 1901-1903, 4(2): 1-83.

Hartman, O. 1964. Polychaeta Errantia of Antarctica. Antarctic Res. Ser., 3: 1-131.

Hartman, O. 1966. Polychaeta Myzostomidae and Sedentaria of Antarctica. Antarctic Res. Ser., 7: 1-158.

Hartman, O. 1967. Polychaeta annelids collected by the USNS Eltanin and Staten Island Cruises, chiefly from Antarctic Seas. Allan Hancock Monogr. Mar. Biol., 2: 1-387.

Hartmann-Schröder, G. 1962. Zur Kenntnis des Eulitorals der chilenischen Pazifikküste und der argentinischen Küste Südpatagoniens unter besonderer Berücksichtigung der Polychaeten und Ostracoden. Tl. II. Die Polychaeten des Eulitorals. Mitt. Hamburg. Zool. Mus. Inst., 60(Suppl.): 57167.

Hartmann-Schröder, G. 1965. Zur Kenntnis des Sublitorals der chilenischen Küste unter besonderer Berücksichtigung der Polychaeten und Ostracoden. Tl. II. Die Polychaeten des Sublitorlas. Mitt. Hamburg. Zool. Mus. Inst., 62(Suppl.): 59-305.

Hartmann-Schröder, G. 1979. Zur Kenntnis des Eulitorals der australischen Küsten unter besonderer Berücksichtigung der Polychaeten und Ostracoden (Teil 2 und Teil 3). Teil 2. Die Polychaeten der tropischen Nordwestküste Australiens (Zwischen Derby im Norden und Port Hedland im Süden). Mitt. Hamb. Zool. Mus. Inst., 76: 75-218.

Hartmann-Schröder, G. 1983. Die Polychaeten der 15., 36. und 76. Reise von FFS "Walter Herwig" zum Patagonischen Schelf (Südwest-Atlantik). Senckenbergiana Marit., 15(4-6): 251-277.

Hartmann-Schröder, G. 1985. Teil 11. Die Polychaeten der antiborealen Südküste Australiens (zwischen Port Lincoln im Westen und Port Augusta im Osten). En: Hartmann-Schröder, G. \& G. Hartmann: Zur Kenntnis des Eulitorals der australischen Küsten unter besonderer Berücksichtigung der Polychaeten und Ostracoden. Mitt. Hamb. Zool. Mus. Inst., 82: 61-99.

Hartmann-Schröder, G. 1986. Die Polychaeten der 56. Reise der "Meteor" zu den South ShetlandInseln (Antarktis). Mitt. Hamb. Zool. Mus. Inst., 83:71-100. 
Hartmann-Schröder, G. \& P. Rosenfeldt. 1989. Die Polychaeten der "Polarstern"-Reise ANT III/2 in die Antarktis 1984. Teil 2: Cirratulidae bis Serpulidae. Mitt. Hamb. Zool. Mus. Inst., 86: 65-106.

Hernández, C.E., R.A. Moreno \& N. Rozbaczylo. 2005. Biogeographical patterns and Rapoport's rule in southeastern Pacific benthic polychaetes of the Chilean coast. Ecography, 28: 363-373.

Hutchings, P.A. \& A. Murray. 1984. Taxonomy of polychaetes from the Hawkesbury River and the Southern estuaries of New South Wales, Australia. Rec. Austral. Mus., 3: 1-118.

Hutchings, P. \& S. Rainer. 1979. The Polychaete fauna of Careel Bay, Pittwater, New South Wales, Australia. J. Nat. Hist., 13: 745-796.

Johnson, H.P. 1901. The Polychaeta of the Puget Sound region. Proc. Boston Soc. Nat. Hist., 29: 381-437.

Jumars, P.A. 1974. A generic revision of the Dorvilleidae (Polychaeta), with six new species from the deep North Pacific. Zool. J. Linn. Soc., 54: 101-135.

Kinberg, J.G.H. 1857. Nya slägten och arter af Annelider. Öfv. Svenska Vetensk. Akad. Förh., 14: 11-14.

Kinberg, J.G.H. 1865. Annulata nova. Öfv. Svenska Vetensk. Akad. Förh., 21: 559-574.

Kinberg, J.G.H. 1858-1910. Annulater. En: Kongliga Svenska Fregatten Eugenies Resa omkring jorden under befäl af C. A. Virgin aren 1851-1853. Vetenskapliga lakttagelser pa Konung Oscar den Förstes befallning utgifna af K. Svenska Vetenskapsakademien. Almquist \& Wiksells, Stockholm, Zoologi, 3: 1-78.

Kinberg, J.G.H. 1867. Annulata nova. Öfv. Svenska Vetensk. Akad. Förh., 23: 337-357.

Knight-Jones, P. \& A.J.M. Walker. 1972. Spirorbinae (Serpulidae: Polychaeta) on limpets from the South Orkney Islands. Bull. Br. Antarct. Surv., 31: 3340.

Kohn, A.J. \& M.C. Lloyd. 1973. Marine polychaete annelids of the Easter Island. Int. Rev. Ges. Hydrobiol., 58(5): 691-712.

Knox, G.A. \& D.B. Cameron. 1998. The marine fauna of the Ross Sea: Polychaeta. NIWA Biodiversity Memoir, 108: 1-125.
Kupriyanova, E. \& I. Jirkov. 1997. Serpulidae (Annelida, Polychaeta) of the Artic Ocean. Sarsia, 82: 203-236.

Lana, P. \& C. S. Bremec. 1994. Sabellariidae (Annelida: Polychaeta) from south America. In: J.-C. Dauvin, L. Laubier \& D. J. Reish (eds.). Actes de la 4ème Conférence Internationale des Polychètes. Mém. Mus. Natn. Hist. Nat., 162: 211-222.

Monro, C.C.A. 1930. Polychaete worms. Discovery Rep., 2: 1-222.

Monro, C.C.A. 1936. Polychaete worms. II. Discovery Rep., 12: 59-198.

Montiel, A., C. Ríos, E. Mutschke \& N. Rozbaczylo. 2004. Poliquetos de fiordos y canales adyacentes al Campo de Hielo Patagónico Sur, Chile (Annelida: Polychaeta). Cienc. Tecnol. Mar, 27(1): 49-67.

Moreno, R.A., C.E. Hernández, M.M. Rivadeneira, M.A. Vidal \& N. Rozbaczylo. 2006. Patterns of endemism in south-eastern Pacific benthic polychaetes of the Chilean coast. J. Biogeogr., 33: 750-759.

Müller, O.F. 1776. Zoologiae Danicae. Prodromus, seu Animalium Daniae et Norvegiae indigenarum characteres, nomina, et synonyma imprimis popularium, $274 \mathrm{pp}$.

Orensanz, J.M. 1974. Los anélidos poliquetos de la Provincia Biogeográfica Argentina. VI. Arabellidae. Physis, 33(87): 381-408.

Orensanz, J.M. 1975. Los anélidos poliquetos de la Provincia Biogeográfica Argentina. VII. Eunicidae y Lysaretidae. Physis, 34(88): 85-111.

Orensanz, J.M. 1976. Los anélidos poliquetos de la Provincia Biogeográfica Magallánica. I. Catálogo de las especies citadas hasta 1974. Laboratorio de Comunidades Bentónicas, Santa Clara del Mar. Contribución Técnica 1: 1-83.

Orensanz, J.M. 1990. The eunicemorph Polychaete annelids from Antarctic and Subantarctic seas. With addenda to the Eunicemorpha of Argentina, Chile, New Zealand, Australia, and the Southern Indian Ocean. Biology of the Antarctic Seas XXI. Antarct. Res. Ser., 52: 1-183.

Petersen, M.E. 1984. Chaetopterus variopedatus (Renier) (Annelida: Polychaeta: Chaetopteridae): a species complex. What species are being used at MBL. Biol. Bull., 167(2): 513. 
Pixell, H. 1913. Polychaeta of families Serpulidae and Sabellidae, collected by Scottish National Antarctic Expedition. Roy. Soc. Edimburgh, Trans., 49(2): 347-358.

Renier, S.A. 1804. Prospetto della Classe dei Vermi.

Rouse, G.W. 2000a. Scolecida. En: P.L. Beesley, G.J.B. Ross \& C.J. Glasby (eds.). Polychaetes and Allies: The Southern Synthesis. Fauna of Australia. Vol. 4A. Polychaeta, Myzostomida, Pogonophora, Echiura, Sipuncula. CSIRO Publishing, Melbourne, p. 62.

Rouse, G.W. 2000b. Sabellida. En: P.L. Beesley, G.J.B. Ross \& C.J. Glasby (eds.). Polychaetes and Allies: The Southern Synthesis. Fauna of Australia. Vol. 4A. Polychaeta, Myzostomida, Pogonophora, Echiura, Sipuncula. CSIRO Publishing, Melbourne, pp. 172-173.

Rouse, G.W. 2000c. Spionida. En: P.L. Beesley, G.J.B. Ross \& C.J. Glasby (eds.). Polychaetes and Allies: The Southern Synthesis. Fauna of Australia. Vol. 4A. Polychaeta, Myzostomida, Pogonophora, Echiura, Sipuncula. CSIRO Publishing, Melbourne, pp. 189-190

Rouse, G.W. \& K. Fauchald. 1997. Cladistics and polychaetes. Zool. Scr., 26: 139-204.

Rouse, G.W. \& F. Pleijel. 2001. Polychaetes. Oxford University Press, Oxford, 354 pp.

Rozbaczylo, N. 1980. Clave para el reconocimiento de familias de anélidos poliquetos del mar chileno. Stud. Neotrop. Fauna Environ., 15(3-4): 167-196.

Rozbaczylo, N. 1985. Los Anélidos Poliquetos de Chile. Indice sinonímico y distribución geográfica de especies. Monografías Biológicas, 3: 1-284.
Rozbaczylo, N., R.A. Moreno \& O. Díaz-Díaz. 2005. Poliquetos bentónicos submareales de fondos blandos de la región de Aysén, Chile: Clado Phyllodocida (Annelida, Polychaeta). Invest. Mar.Valparaíso, 33(1): 69-89.

Rozbaczylo, N., R.A. Moreno, O. Díaz-Díaz \& S. Martínez. 2006. Poliquetos bentónicos submareales de fondos blandos de la región de Aysén, Chile: Clado Terebellida (Annelida, Polychaeta). Cienc. Tecnol. Mar 29(1): en prensa.

Schmarda, L.K. 1861. Neue wirbellose Thiere beobachtet und gesammelt auf einer Reise um die Erde 1853 bis 1857. 1: Turbellarien, Rotatorien und Anneliden, 2: 1-164.

Ten Hove, H. \& P. Wolf. 1984. Serpulidae. En: J.M. Uebelacker \& P.G. Johnson (eds.). Taxonomic Guide to the Polychaetes of Northern Gulf of Mexico. B.A. Vittor \& Ass. Inc. Mobile Alabama. Vol. 7(55): 1-34.

Vine, P.J. 1977. The marine fauna of New Zealand: Spirorbinae (Polychaeta: Serpulidae). Mem. New Zeland Oceanogr. Inst., 68: 7-68.

Wesenberg-Lund, E. 1962. Polychaeta Errantia. Reports of the Lund University Chile Expedition 1948-1949, (43). Lunds Universitets Årsskrift. N.F. Avd. 2, 57(12): 1-139.

Zibrowius, H.W. 1968. Étude morphologique, systématique et écologique, des Serpulidae (Annelida: Polychaeta) de la région de Marseille. Rec. Trav. St. Mar. End., Bull., 43(59): 81-252. 